\title{
Synthesis of Analogues of Gingerol and Shogaol, the Active Pungent Principles from the Rhizomes of Zingiber officinale and Evaluation of Their Anti-Platelet Aggregation Effects
}

\author{
Hung-Cheng Shih ${ }^{1, \dagger}$, Ching-Yuh Chern ${ }^{2, \dagger}$, Ping-Chung Kuo ${ }^{3}$, You-Cheng Wu ${ }^{1}$, Yu-Yi Chan ${ }^{4}$,
} Yu-Ren Liao ${ }^{1}$, Che-Ming Teng ${ }^{5}$ and Tian-Shung $\mathrm{Wu}^{1}{ }^{1, *}$

1 Department of Chemistry, National Cheng Kung University, Tainan 701, Taiwan; E-Mails: 13894106@mail.ncku.edu.tw (H.-C.S.); hope.wu@ge.com (Y.-C.W.); truthloveroy@yahoo.com.tw (Y.-R.L.)

2 Department of Applied Chemistry, National Chiayi University, Chiayi 600, Taiwan; E-Mail: cychern@mail.ncyu.edu.tw

3 Department of Biotechnology, National Formosa University, Yunlin 632, Taiwan; E-Mail: pcckuoo@nfu.edu.tw

4 Department of Biotechnology, Southern Taiwan University, Tainan 710, Taiwan; E-Mail: yuyichan@mail.stust.edu.tw

5 College of Medicine, Pharmacological Institute, National Taiwan University, Taipei 100, Taiwan; E-Mail: cmteng@ntu.edu.tw

$\dagger$ These authors contributed equally to this work.

* Author to whom correspondence should be addressed; E-Mail: tswu@mail.ncku.edu.tw; Tel./Fax: +886-6-2747538.

Received: 2 January 2014; in revised form: 17 February 2014 / Accepted: 21 February 2014 / Published: 4 March 2014

\begin{abstract}
The present study was aimed at discovering novel biologically active compounds based on the skeletons of gingerol and shogaol, the pungent principles from the rhizomes of Zingiber officinale. Therefore, eight groups of analogues were synthesized and examined for their inhibitory activities of platelet aggregation induced by arachidonic acid, collagen, platelet activating factor, and thrombin. Among the tested compounds, [6]-paradol (5b) exhibited the most significant anti-platelet aggregation activity. It was the most potent candidate, which could be used in further investigation to explore new drug leads.
\end{abstract}


Keywords: Zingiber officinale; ginger; gingerol; shogaol; anti-platelet aggregation

\section{Introduction}

Ginger (Chinese name: Shengjiang), derived from the rhizomes of Zingiber officinale Roscoe, is a well-known spice and is most frequently prescribed as a traditional Chinese medicine for its stomachic, antiemetic, antidiarrheal, expectorant, antiasthmatic, hemostatic and cardiologic properties for the treatment of several gastrointestinal and respiratory diseases [1-3]. The most famous traditional medicinal application of $Z$. officinale is to promote blood circulation for the removal of blood stasis, a mechanism that is related to anti-platelet aggregation activity [4,5]. Numerous chemical investigations of the pungent and bioactive principles of ginger have been carried out [6-19]. The pungent principles reported from the rhizomes of Zingiber officinale include: zingerone, gingerols, gingerdiols, gingerdiones, and shogaols (Figure 1).

Figure 1. Chemical structures of the pungent principles from the rhizomes of Zingiber officinale.

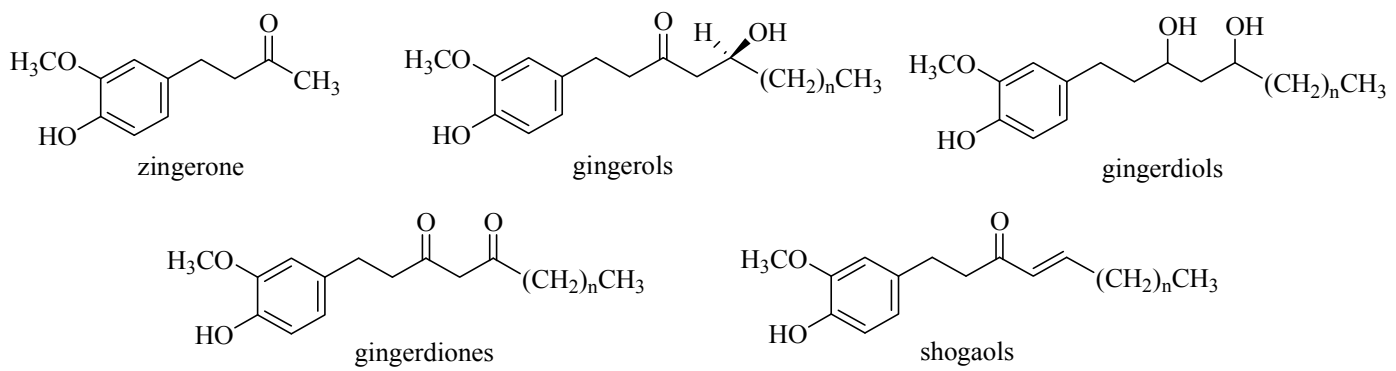

In the course of our continuing research program aimed at discovering novel bioactive constituents from natural sources, thrombolytic and vasoactive activity examinations were carried out, and the ether extracts of the rhizomes of $Z$. officinale were found to exhibit significant anti-platelet aggregation activity and vasorelaxing effects. In our previous article [20], twenty-nine compounds were identified, and [6]-gingerol and [6]-shogaol exhibited potent anti-platelet aggregation bioactivity. These results initiated our interest in searching for more potent antiplatelet aggregation agents from the analogues of gingerol and shogaol. Therefore, in the present study eight groups of compounds (Figure 2) were prepared and subjected to examinations of their anti-platelet aggregation activity.

Figure 2. Chemical structures of the synthetic analogues of gingerol and shogaol.

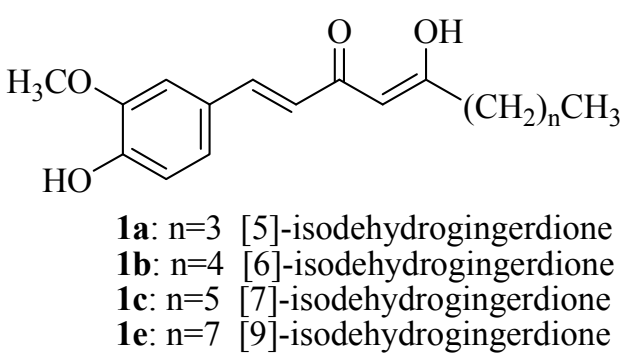

1a: $\mathrm{n}=3$ [5]-isodehydrogingerdione

1c: $n=5$ [7]-isodehydrogingerdione

1e: $n=7$ [9]-isodehydrogingerdione

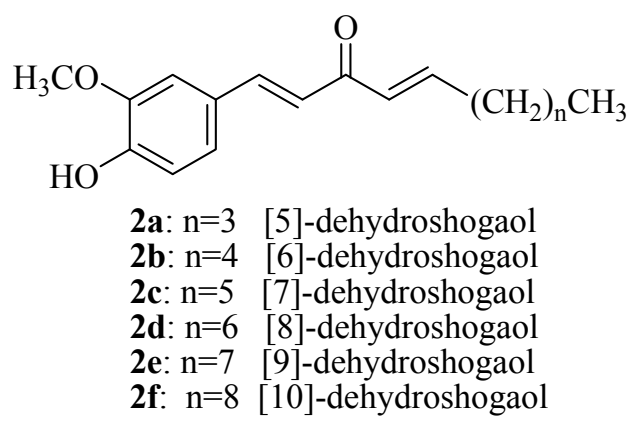


Figure 2. Cont.

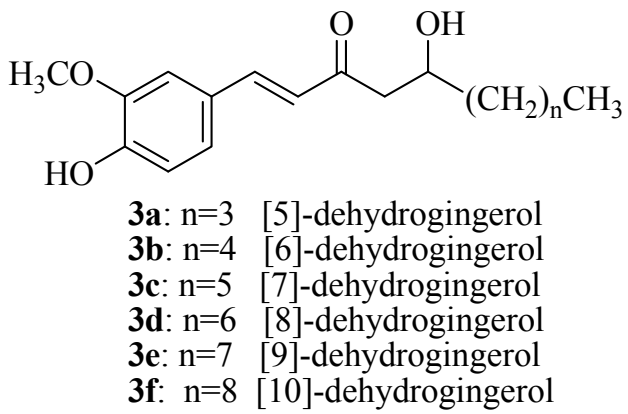<smiles>CCCCCC(=O)CCc1ccc(O)c(OC)c1</smiles>

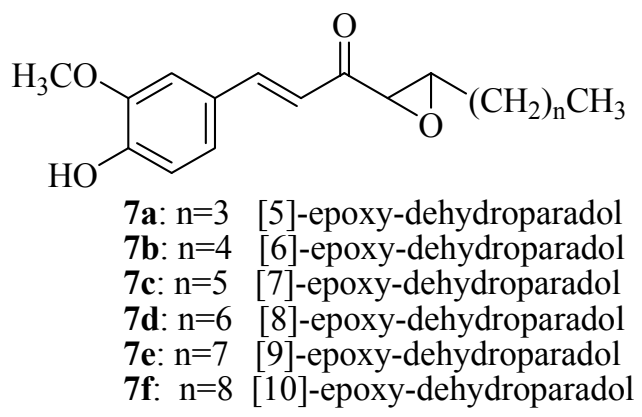<smiles>CCCC=CC(=O)CCc1ccc(O)c(OC)c1</smiles><smiles>CCCCCCC(=O)/C=C/c1ccc(O)c(OC)c1</smiles>
6a: $n=3$ [5]-dehydroparadol
6b: $\mathrm{n}=4$ [6]-dehydroparadol
6c: $\mathrm{n}=5$ [7]-dehydroparadol
6d: $\mathrm{n}=6$ [8]-dehydroparadol
6e: $n=7$ [9]-dehydroparadol
6f: $n=8$ [10]-dehydroparadol

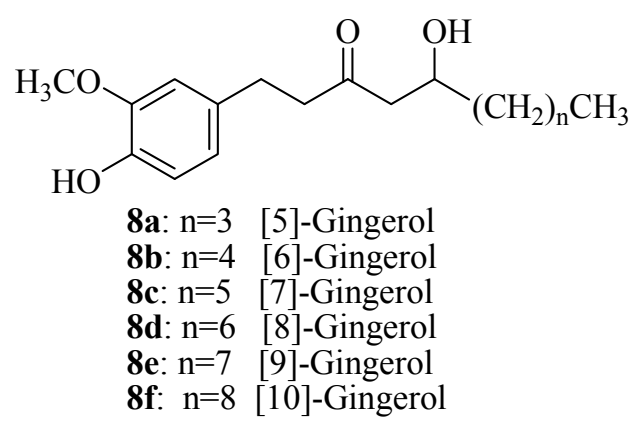

\section{Results and Discussion}

\subsection{Chemistry}

At first, the dehydrozingerone 9 was prepared by vanillin condensation with a good yield (89\%), Equation (1). Then the cross aldol condensations of $\alpha, \beta$-unsaturated ketone 9 with different aldehydes were investigated using various bases as catalysts. The major products were the dehydrogingerols $\mathbf{3 a}-\mathbf{f}$, and the minor products dehydroshogaols $2 \mathbf{a}-\mathbf{f}$ were obtained in the optimum yield (6\%-15\%) when lithium bis(trimethylsilyl)amide (LiHMDS) was employed. Therefore, deprotonation of 9 with LiHMDS in tetrahydrofuran at $0{ }^{\circ} \mathrm{C}$ and subsequent trapping with aldehydes Equation (2) afforded products $\mathbf{2 a}-\mathbf{f}$ and $\mathbf{3 a}-\mathbf{f}$ with moderate yields in a range between $50 \%$ and $66 \%$ (Table 1).<smiles>CCOc1ccc(O)c(OCCNC(=O)c2ccc(O)c(OC)c2)c1</smiles> 


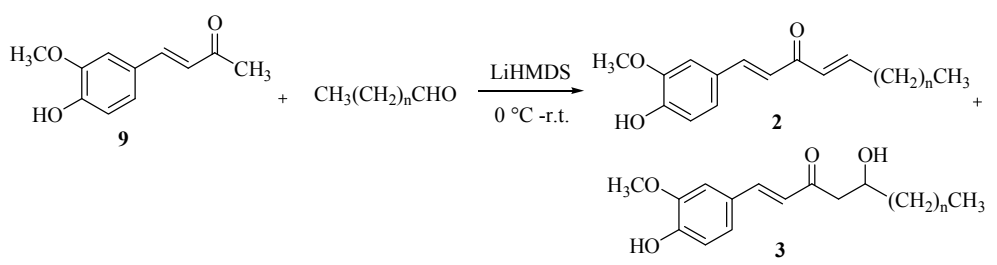

Table 1. The yields of products $\mathbf{2}$ and $\mathbf{3}$ from cross aldol condensation.

\begin{tabular}{ccc}
\hline$[\boldsymbol{n}]$ & 2 yield\% & 3 yield\% \\
\hline a: $n=5$ & 9 & 65 \\
b: $n=6$ & 15 & 59 \\
c: $n=7$ & 13 & 56 \\
d: $n=8$ & 15 & 66 \\
e: $n=9$ & 13 & 58 \\
f: $n=10$ & 6 & 50 \\
\hline
\end{tabular}

However, similar reaction conditions under air atmosphere furnished low yields of [n]-epoxy-dehydroparadols 7a-f (Equation (3), Table 2), and comparatively, relatively higher yields of dehydroshogaols $\mathbf{2 a}-\mathbf{f}(15 \%-21 \%)$.

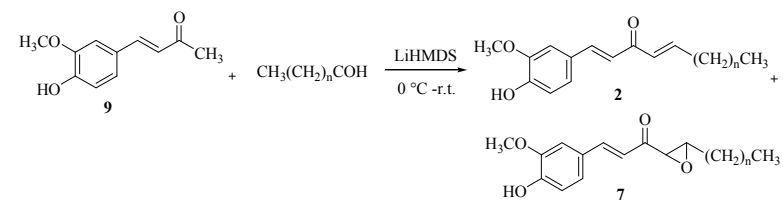

Table 2. Cross aldol condensation under air atmosphere.

\begin{tabular}{ccc}
\hline$[\boldsymbol{n}]$ & $\mathbf{2}$ yield\% & $\mathbf{7}$ yield \% \\
\hline a: $n=5$ & 19 & 8 \\
b: $n=6$ & 21 & 10 \\
c: $n=7$ & 18 & 9 \\
d: $n=8$ & 18 & 9 \\
e: $n=9$ & 16 & 8 \\
f: $n=10$ & 15 & 8 \\
\hline
\end{tabular}

Chlorination and dehydrohalogenation of alcohols $3 \mathbf{a}-\mathbf{f}$ with $\mathrm{HCl}$ and $\mathrm{K}_{2} \mathrm{CO}_{3}$, respectively, produced quantitative yields of adducts $\mathbf{2 a}-\mathbf{f}$ Equation (4) and reduced the occurrence of trace amounts of 10. The catalytic hydrogenation of $[n]$-dehydroshogaols $\mathbf{2 a - f}$ over palladium on charcoal afforded $[n]$-paradols 5 and trace amounts of secondary alcohol 11. It was surprising that $[n]$-dehydroparadols 6 could be obtained with the same method only reduced the amount of palladium on charcoal from 0.05 to 0.015 eq. The results are shown in Equation (5) and Table 3.

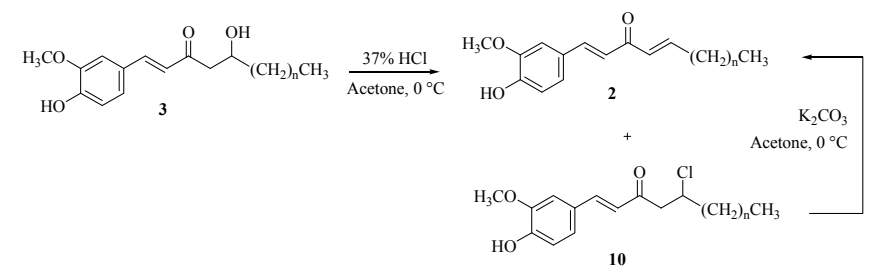




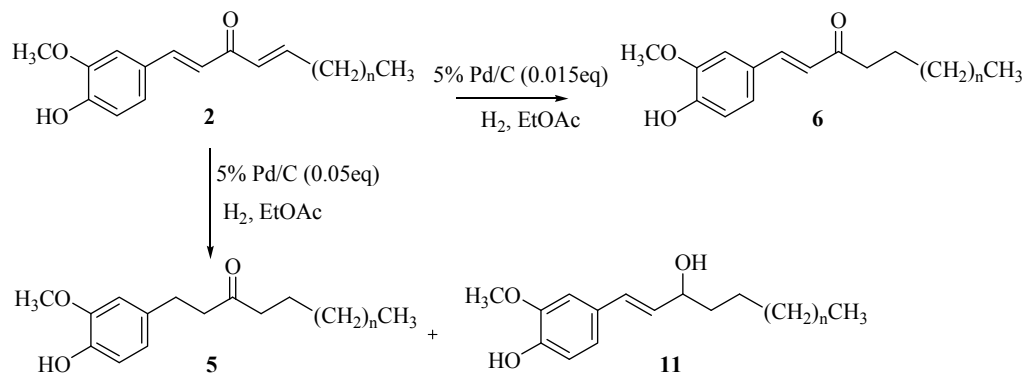

Table 3. Hydrogenation of compound 2 to afford product 5 and 6.

\begin{tabular}{ccc}
\hline$[\boldsymbol{n}]$ & $\mathbf{5}$ yield\% & $\mathbf{6}$ yield \% \\
\hline a: $n=5$ & 79 & 80 \\
b: $n=6$ & 78 & 76 \\
c: $n=7$ & 81 & 75 \\
d: $n=8$ & 77 & 73 \\
e: $n=9$ & 80 & 74 \\
f: $n=10$ & 79 & 79 \\
\hline
\end{tabular}

The same hydrogenation procedure was applied in $[n]$-dehydrogingerols $\mathbf{3}$, and a high yield of $[n]$-gingerols 8 was obtained ( $83 \%-86 \%$ ). Dehydration of 8 with $\mathrm{HCl} / \mathrm{K}_{2} \mathrm{CO}_{3}$ gave approximately $85 \%$ of $[n]$-shogaols 4 (Equation (6), Table 4). Although there were many reagents available for the oxidation of secondary alcohols to ketone, unfortunately, most of these oxidizing agents did not show sufficient activity except in the case of Swern oxidation, which yielded a moderate amount of oxidized compound 1 (Equation (7), Table 5).

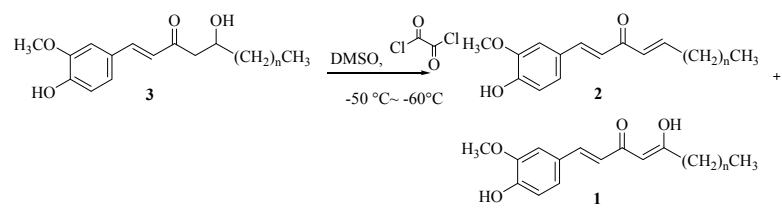

Table 4. Hydrogenation and elimination of compound 3 to yield products 8 and 4 .

\begin{tabular}{ccc}
\hline$[\boldsymbol{n}]$ & $\mathbf{8}$ yield\% & 4 yield\% \\
\hline a: $n=5$ & 85 & 86 \\
$\mathbf{b}: n=6$ & 84 & 85 \\
$\mathbf{c}: n=7$ & 86 & 83 \\
$\mathbf{d}: n=8$ & 83 & 79 \\
e: $n=9$ & 84 & 85 \\
f: $n=10$ & 85 & 88 \\
\hline
\end{tabular}


Table 5. The Swern oxidation of compound $\mathbf{3}$ to obtain products $\mathbf{2}$ and $\mathbf{1 .}$

\begin{tabular}{ccc}
\hline$[\boldsymbol{n}]$ & 2 yield\% & 1 yield\% \\
\hline a: $n=5$ & 16 & 51 \\
b: $n=6$ & 15 & 49 \\
c: $n=7$ & 11 & 59 \\
e: $n=9$ & 17 & 48 \\
\hline
\end{tabular}

\subsection{Anti-Platelet Aggregation Evaluation Bioassay}

Platelets circulate in the blood of mammals and are involved in hemostasis, leading to the formation of blood clots. Too many platelets form blood clots that may obstruct blood vessels and induce strokes, myocardial infarctions, and pulmonary embolisms. Sometimes this situation also results in the blockage of blood vessels to other parts of the body, including the extremities of the arms or legs [21]. The traditional medicinal use of ginger is to promote the blood circulation necessary for removing blood stasis. Therefore, synthetic derivatives were examined in the anti-platelet aggregation bioassay to test for the presence of activity. The anti-platelet aggregation results are summarized in Tables 6-13. All the tested compounds displayed significant inhibitory effects on the aggregation of washed rabbit platelets stimulated by arachidonic acid (AA). At a $10 \mu \mathrm{g} / \mathrm{mL}$ concentration, most of the tested compounds with the exception of $\mathbf{3 a}, \mathbf{3 d}$, and 7e caused the inhibition percentages of aggregation induced by AA $(100 \mu \mathrm{M})$ to be higher than $90 \%$. On the other hand, the activities of these synthetic derivatives against platelet activating factor (PAF) and thrombin (Thr) induced aggregation were insignificant.

Among these derivatives, the $[n]$-paradols (5a-f) series were the most active compounds, and [6]-paradol 5b displayed the most significant inhibition, with an $I C_{50}$ value of $70 \mathrm{ng} / \mathrm{mL}$ (Table 6). $[n]$-Dehydroparadols $(\mathbf{6 a}-\mathbf{f})$ were generally less potent than the corresponding $[n]$-paradols $(\mathbf{5 a}-\mathbf{f})$ derivatives (Table 7). The most potent compound was [10]-dehydroparadol $6 \mathbf{f}(n=8)$, with an $I C_{50}$ value of $160 \mathrm{ng} / \mathrm{mL}$, which was a 2.3 -fold decrease in activity due to the introduction of an unsaturated $\mathrm{C}=\mathrm{C}$ bond. The $[n]$-shogaols $(\mathbf{4 a}-\mathbf{f})$ series (Table 8$)$ also displayed weaker inhibition of aggregation induced by AA $(100 \mu \mathrm{M})$ compared to their related $[n]$-paradol derivatives $\mathbf{5 a}-\mathbf{f}$; however, they were more active than the $[n]$-dehydroparadols $(\mathbf{6 a}-\mathbf{f})$ series. It was evident that introduction of unsaturated $\mathrm{C}=\mathrm{C}$ bonds would decrease the inhibitory activity. But the location of unsaturated $\mathrm{C}=\mathrm{C}$ bonds also influences the inhibitory activity. The $[n]$-dehydroshogaols (2a-f) (Table 9) which possess one more $\alpha, \beta$-unsaturation $\mathrm{C}=\mathrm{C}$ bond exhibited further decreased inhibitory activity. However, their inhibition aggregation potency induced by collagen (Col) was generally more significant than their $[n]$-paradol counterparts 5a-f. Among the $[n]$-shogaols (4a-f) and $[n]$-dehydroshogaols (2a-f), [10]-shogaols (4f) $(n=8)$ exhibited the most significant inhibition of aggregation induced by Col $(10 \mu \mathrm{M})$, with an $I C_{50}$ value lower than $5 \mu \mathrm{g} / \mathrm{mL}$. The possible mechanism was one in which the rigid styryl carbonyl ethylene prevented the alkyl tail from turning sideways, where a putative hydrophobic pocket may have been located. Therefore, a free alkyl chain could overcome such an effect. 
Table 6. Antiplatelet activity of $[n]$-paradols $\mathbf{5 a}-\mathbf{f}$.

\begin{tabular}{|c|c|c|c|c|c|c|c|c|}
\hline \multicolumn{9}{|c|}{ Inhibition (\%) } \\
\hline Inducer & Control & Conc. $(\mu \mathrm{g} / \mathrm{mL})$ & $5 \mathbf{5 a}$ & $5 \mathbf{5 b}$ & $5 \mathrm{c}$ & $5 d$ & $5 e$ & $5 f$ \\
\hline \multirow{10}{*}{ AA $(100 \mu \mathrm{M})$} & \multirow{10}{*}{$0.0 \pm 1.3$} & 10 & $100.0 \pm 1.3 * * *$ & $100.0 \pm 1.3 * * *$ & $100.0 \pm 1.3^{* * *}$ & $100.0 \pm 1.3^{* * *}$ & $100.0 \pm 0.4$ & $100.0 \pm 0.4 * * *$ \\
\hline & & 5 & - & - & $100.0 \pm 1.3 * * *$ & $100.0 \pm 1.3 * * *$ & - & $100.0 \pm 0.4 * * *$ \\
\hline & & 2 & - & $100.0 \pm 1.3 * * *$ & $94.9 \pm 3.4 * * *$ & $98.4 \pm 0.2 * * *$ & $100.0 \pm 0.4 * * *$ & $87.4 \pm 4.8 * * *$ \\
\hline & & 1 & $100.0 \pm 1.3 * * *$ & $97.0 \pm 1.4 * * *$ & $93.2 \pm 5.0 * * *$ & $90.9 \pm 4.0 * * *$ & $96.9 \pm 2.1 * * *$ & $75.9 \pm 4.7 * * *$ \\
\hline & & 0.5 & $87.1 \pm 6.5^{* * *}$ & $92.8 \pm 5.4 * * *$ & $90.0 \pm 7.9 * * *$ & $81.6 \pm 9.4 * * *$ & $89.8 \pm 3.8 * * *$ & $53.6 \pm 2.1 * * *$ \\
\hline & & 0.2 & $63.9 \pm 10.6^{* * *}$ & $79.9 \pm 8.9 * * *$ & $76.0 \pm 10.7 * * *$ & $76.9 \pm 12.1 * * *$ & $21.7 \pm 3.4 * * *$ & $26.4 \pm 6.1 * * *$ \\
\hline & & 0.1 & $57.3 \pm 12.9 * * *$ & $67.6 \pm 13.0 * * *$ & $68.0 \pm 1.7^{* * *}$ & $53.0 \pm 13.7 * *$ & - & $6.4 \pm 2.2 * *$ \\
\hline & & 0.05 & $29.0 \pm 13.6$ & $52.6 \pm 13.1 * * *$ & $17.0 \pm 5.0 *$ & $26.7 \pm 8.9 *$ & - & - \\
\hline & & 0.02 & $10.0 \pm 3.5 *$ & $14.7 \pm 5.6$ & $7.0 \pm 3.9$ & $8.1 \pm 2.9$ & - & - \\
\hline & & 0.01 & $-0.9 \pm 0.0$ & $5.5 \pm 1.4$ & $2.4 \pm 1.7$ & - & - & - \\
\hline$I C_{50}(\mu \mathrm{g} / \mathrm{mL})$ & - & - & 0.10 & 0.07 & 0.12 & 0.12 & 0.30 & 0.49 \\
\hline Col $(10 \mu \mathrm{M})$ & $0.0 \pm 0.8$ & 10 & $61.7 \pm 3.8 * * *$ & $33.3 \pm 4.1 * * *$ & $39.7 \pm 3.8 * * *$ & $52.7 \pm 3.5 * * *$ & $36.9 \pm 5.4 * * *$ & $32.9 \pm 3.1 * * *$ \\
\hline PAF (2 ng/mL) & $0.0 \pm 1.0$ & 10 & $1.9 \pm 0.8 * * *$ & $1.9 \pm 0.3$ & $0.7 \pm 0.1$ & $1.3 \pm 0.0$ & $-0.8 \pm 0.4$ & $21 \pm 1.6$ \\
\hline $\operatorname{Thr}(0.1 \mathrm{U} / \mathrm{mL})$ & $0.0 \pm 1.0$ & 10 & $-2.1 \pm 0.2 *$ & $0.0 \pm 0.6$ & $-2.7 \pm 0.1$ & $-1.0 \pm 0.2$ & $0.2 \pm 0.4$ & $2.5 \pm 0.4$ \\
\hline
\end{tabular}

Table 7. Antiplatelet activity of $[n]$-dehydroparadols $\mathbf{6 a}-\mathbf{f}$.

\begin{tabular}{|c|c|c|c|c|c|c|c|c|}
\hline \multicolumn{9}{|c|}{ Inhibition (\%) } \\
\hline Inducer & Control & Conc. $(\mu \mathrm{g} / \mathrm{mL})$ & $\mathbf{6 a}$ & $6 b$ & $6 c$ & 6d & $6 e$ & $6 f$ \\
\hline \multirow{8}{*}{ AA $(100 \mu \mathrm{M})$} & \multirow{8}{*}{$0.0 \pm 0.4$} & 10 & $100.0 \pm 0.4 * * *$ & $100.0 \pm 0.4$ & $100.0 \pm 0.4 * * *$ & $100.0 \pm 0.4 * * *$ & $100.0 \pm 0.4 * * *$ & $100.0 \pm 0.4 * * *$ \\
\hline & & 5 & - & $100.0 \pm 0.4$ & $97.7 \pm 1.6 * * *$ & $98.4 \pm 0.9 * * *$ & - & - \\
\hline & & 2 & $100.0 \pm 0.4 * * *$ & $94.9 \pm 4.0$ & $96.6 \pm 2.5 * * *$ & $93.1 \pm 5.1 * * *$ & $100.0 \pm 0.4 * * *$ & $100.0 \pm 0.4 * * *$ \\
\hline & & 1 & $78.4 \pm 8.5 * * *$ & $64.9 \pm 10.7$ & $86.3 \pm 7.4 * * *$ & $73.1 \pm 1.0 * * *$ & $75.2 \pm 7.8 * * *$ & $97.7 \pm 1.6 * * *$ \\
\hline & & 0.5 & $64.2 \pm 9.1 * * *$ & $50.4 \pm 7.2$ & $59.4 \pm 7.0 * * *$ & $62.3 \pm 0.7 * * *$ & $55.2 \pm 11.6 * * *$ & $97.7 \pm 1.6 * * *$ \\
\hline & & 0.2 & $38.9 \pm 10.8 * *$ & $14.5 \pm 4.8$ & $40.8 \pm 4.0 * * *$ & $36.3 \pm 0.0 * * *$ & $8.7 \pm 1.0 * * *$ & $79.4 \pm 7.7 * * *$ \\
\hline & & 0.1 & $14.4 \pm 6.5$ & $6.7 \pm 3.2$ & $16.1 \pm 1.9 * * *$ & $12.2 \pm 3.1 *$ & - & $31.4 \pm 8.6^{*}$ \\
\hline & & 0.05 & $3.4 \pm 0.9$ & - & $6.7 \pm 1.3 *$ & - & - & $5.4 \pm 1.9 *$ \\
\hline$I C_{50}(\mu \mathrm{g} / \mathrm{mL})$ & - & - & 0.32 & 0.56 & 0.35 & 0.40 & 0.52 & 0.16 \\
\hline $\mathrm{Col}(10 \mu \mathrm{M})$ & $0.0 \pm 1.0$ & 10 & $42.8 \pm 4.7 * * *$ & $39.3 \pm 4.6 * * *$ & $18.4 \pm 1.6^{* * *}$ & $36.1 \pm 0.1 *$ & $20.2 \pm 2.6 * * *$ & $18.9 \pm 3.4^{* * *}$ \\
\hline PAF (2 ng/mL) & $0.0 \pm 1.0$ & 10 & $5.3 \pm 3.3$ & $0.4 \pm 0.2$ & $0.5 \pm 0.5$ & $2.0 \pm 2.5$ & $1.1 \pm 0.3$ & $-1.0 \pm 0.5$ \\
\hline $\operatorname{Thr}(0.1 \mathrm{U} / \mathrm{mL})$ & $0.0 \pm 0.9$ & 10 & $4.3 \pm 0.1 * *$ & $1.8 \pm 0.6$ & $3.0 \pm 1.6$ & $1.5 \pm 0.5$ & $5.0 \pm 0.1$ & $0.9 \pm 0.5$ \\
\hline
\end{tabular}


Table 8. Antiplatelet activity of $[n]$-shogaols $\mathbf{4 a}-\mathbf{f}$.

\begin{tabular}{|c|c|c|c|c|c|c|c|c|}
\hline \multicolumn{9}{|c|}{ Inhibition (\%) } \\
\hline Inducer & Control & Conc. $(\mu \mathrm{g} / \mathrm{mL})$ & $\mathbf{4 a}$ & $\mathbf{4 b}$ & $4 c$ & $\mathbf{4 d}$ & $4 e$ & $\mathbf{4 f}$ \\
\hline \multirow{7}{*}{$\mathrm{AA}(100 \mu \mathrm{M})$} & \multirow{7}{*}{$0.0 \pm 1.2$} & 10 & $100.0 \pm 1.2 * * *$ & $100.0 \pm 1.2 * * *$ & $100.0 \pm 1.2 * * *$ & $100.0 \pm 1.2 * * *$ & $100.0 \pm 1.2 * * *$ & $100.0 \pm 1.2 * * *$ \\
\hline & & 1 & $100.0 \pm 1.2 * * *$ & $100.0 \pm 1.2 * * *$ & - & - & - & $100.0 \pm 1.2 * * *$ \\
\hline & & 0.5 & $70.0 \pm 19.6 * * *$ & $94.2 \pm 3.4 * * *$ & $100.0 \pm 1.2 * * *$ & $100.0 \pm 1.2 * * *$ & $100.0 \pm 1.2 * * *$ & $83.1 \pm 6.0 * * *$ \\
\hline & & 0.2 & $52.8 \pm 22.1 * *$ & $78.0 \pm 15.2 * * *$ & $62.3 \pm 18.1 * *$ & $74.9 \pm 14.3 * * *$ & $57.9 \pm 1.1 * * *$ & $81.2 \pm 9.1 * * *$ \\
\hline & & 0.1 & $14.7 \pm 8.3$ & $47.3 \pm 19.6 *$ & $54.1 \pm 21.7 *$ & $14.3 \pm 16.0 * *$ & $36.0 \pm 14.4 *$ & $41.8 \pm 17.8 *$ \\
\hline & & 0.05 & $5.3 \pm 2.7$ & $13.7 \pm 5.4 *$ & $7.9 \pm 2.7$ & $5.2 \pm 2.2$ & $7.2 \pm 2.7$ & $30.9 \pm 21.5$ \\
\hline & & 0.02 & - & $2.8 \pm 1.1$ & - & - & - & $2.8 \pm 0.2$ \\
\hline$I C_{50}(\mu \mathrm{g} / \mathrm{mL})$ & - & - & 0.23 & 0.12 & 0.13 & 0.15 & 0.15 & 0.11 \\
\hline \multirow{5}{*}{$\operatorname{Col}(10 \mu \mathrm{M})$} & \multirow{5}{*}{$0.0 \pm 1.7$} & 10 & $91.2 \pm 6.0 * * *$ & $84.5 \pm 11.9 * * *$ & $78.7 \pm 13.2 * * *$ & $74.5 \pm 14.0 * * *$ & $79.3 \pm 16.2 * * *$ & $91.1 \pm 1.9 * * *$ \\
\hline & & 5 & $55.3 \pm 7.7 * * *$ & $50.1 \pm 5.4 * * *$ & $41.4 \pm 4.3 * * *$ & $38.0 \pm 7.9 * * *$ & $45.5 \pm 14.7 * *$ & $82.8 \pm 7.3 * * *$ \\
\hline & & 2 & $20.0 \pm 6.6^{*}$ & $13.3 \pm 2.5 * *$ & $9.8 \pm 0.6 * *$ & $6.0 \pm 1.2$ & $27.0 \pm 15.6$ & $36.3 \pm 14.9 *$ \\
\hline & & 1 & $1.5 \pm 0.7$ & $2.5 \pm 2.5$ & $1.7 \pm 0.8$ & - & $4.0 \pm 2.0$ & $11.3 \pm 3.3 *$ \\
\hline & & 0.5 & - & - & - & - & - & $3.2 \pm 0.7$ \\
\hline PAF (2 ng/mL) & $0.0 \pm 1.2$ & 10 & $9.3 \pm 0.1 * * *$ & $6.4 \pm 0.7 *$ & $6.0 \pm 1.3$ & $5.2 \pm 1.1$ & $4.5 \pm 2.1$ & $6.6 \pm 2.2$ \\
\hline Thr $(0.1 \mathrm{U} / \mathrm{mL})$ & $0.0 \pm 0.4$ & 10 & $3.5 \pm 0.6 *$ & $1.7 \pm 0.2$ & $1.5 \pm 0.2$ & $2.2 \pm 0.6$ & $1.0 \pm 1.0$ & $2.2 \pm 3.4$ \\
\hline
\end{tabular}


Table 9. Antiplatelet activity of $[n]$-dehydroshogaols $\mathbf{2 a}-\mathbf{f}$.

\begin{tabular}{|c|c|c|c|c|c|c|c|c|}
\hline \multicolumn{9}{|c|}{ Inhibition (\%) } \\
\hline Inducer & Control & Conc. $(\mu \mathrm{g} / \mathrm{mL})$ & $\mathbf{2 a}$ & $2 \mathbf{b}$ & $2 c$ & $2 d$ & $2 \mathbf{e}$ & $2 f$ \\
\hline \multirow{7}{*}{ AA $(100 \mu \mathrm{M})$} & \multirow{7}{*}{$0.0 \pm 1.4$} & 10 & $100.0 \pm 1.4 * * *$ & $100.0 \pm 1.4 * * *$ & $100.0 \pm 1.4 * * *$ & $100.0 \pm 1.4 * * *$ & $100.0 \pm 1.4 * * *$ & $100.0 \pm 1.4 * * *$ \\
\hline & & 5 & $100.0 \pm 1.4 * * *$ & $100.0 \pm 1.4 * * *$ & $100.0 \pm 1.4 * * *$ & $100.0 \pm 1.4 * * *$ & $100.0 \pm 1.4 * * *$ & $96.9 \pm 1.1 * * *$ \\
\hline & & 2 & $86.4 \pm 7.0 * * *$ & $71.1 \pm 13.0 * * *$ & $84.1 \pm 12.4 * * *$ & $86.4 \pm 9.7 * * *$ & $97.7 \pm 0.6 * * *$ & $95.5 \pm 2.3 * * *$ \\
\hline & & 1 & $35.6 \pm 12.4 * * *$ & $30.7 \pm 8.6 * *$ & $61.4 \pm 18.1 * *$ & $54.5 \pm 13.4 * * *$ & $91.1 \pm 6.4 * * *$ & $81.4 \pm 7.7 * * *$ \\
\hline & & 0.5 & $26.5 \pm 18.4$ & $19.7 \pm 12.9$ & $19.2 \pm 8.1 *$ & $9.2 \pm 0.3 * * *$ & $31.7 \pm 18.3$ & $60.5 \pm 19.6 * *$ \\
\hline & & 0.2 & $7.2 \pm 3.5$ & $3.3 \pm 0.5$ & $5.6 \pm 0.9 *$ & $1.5 \pm 0.5$ & $8.6 \pm 4.2$ & $3.3 \pm 1.5$ \\
\hline & & 0.1 & - & - & - & - & $2.1 \pm 0.9$ & - \\
\hline$I C_{50}(\mu \mathrm{g} / \mathrm{mL})$ & - & - & 0.96 & 1.18 & 0.88 & 0.99 & 0.57 & 0.51 \\
\hline \multirow{4}{*}{$\operatorname{Col}(10 \mu \mathrm{M})$} & \multirow{4}{*}{$0.0 \pm 0.6$} & 10 & $93.3 \pm 5.3 * * *$ & $26.9 \pm 9.2 *$ & $66.8 \pm 14.1 * * *$ & $51.6 \pm 14.8 * *$ & $44.8 \pm 7.8 * * *$ & $62.1 \pm 13.9 * * *$ \\
\hline & & 5 & $29.1 \pm 8.0 * *$ & $2.1 \pm 0.9$ & $7.1 \pm 1.5 * *$ & $8.3 \pm 1.3 * * *$ & - & - \\
\hline & & 2 & $3.5 \pm 1.1$ & - & - & - & - & - \\
\hline & & 0.2 & $92.2 \pm 2.2 * * *$ & - & - & - & - & - \\
\hline PAF (2 ng/mL) & $0.0 \pm 1.3$ & 10 & $11.2 \pm 1.4 * *$ & $5.0 \pm 3.1$ & $6.8 \pm 0.7 *$ & $5.9 \pm 0.7 *$ & $5.9 \pm 1.0 *$ & $5.6 \pm 0.7 *$ \\
\hline $\operatorname{Thr}(0.1 \mathrm{U} / \mathrm{mL})$ & $0.0 \pm 0.4$ & 10 & $5.1 \pm 2.1$ & $1.6 \pm 1.4$ & $0.9 \pm 1.3$ & $1.8 \pm 1.6$ & $3.2 \pm 1.2$ & $1.0 \pm 1.3$ \\
\hline
\end{tabular}


Table 10. Antiplatelet activity of $[n]$-gingerols $\mathbf{8 a}-\mathbf{f}$.

\begin{tabular}{|c|c|c|c|c|c|c|c|c|}
\hline \multicolumn{9}{|c|}{ Inhibition (\%) } \\
\hline Inducer & Control & Conc. $(\mu \mathrm{g} / \mathrm{mL})$ & $8 a$ & $8 b$ & $8 c$ & $8 d$ & $8 e$ & $8 f$ \\
\hline \multirow[t]{8}{*}{ AA $(100 \mu \mathrm{M})$} & $0.0 \pm 1.3$ & 10 & $93.0 \pm 2.6 * * *$ & $100.0 \pm 1.3 * * *$ & $93.8 \pm 4.2 * * *$ & $96.3 \pm 1.1 * * *$ & $100.0 \pm 1.3 * * *$ & $100.0 \pm 1.3 * * *$ \\
\hline & & 5 & $59.9 \pm 13.4 * * *$ & $100.0 \pm 1.3 * * *$ & $79.6 \pm 10.5 * * *$ & $90.4 \pm 4.3 * * *$ & $97.0 \pm 1.4 * * *$ & $99.2 \pm 0.5 * * *$ \\
\hline & & 2 & $37.4 \pm 15.2 *$ & $75.6 \pm 9.7 * * *$ & $74.8 \pm 12.9$ *** & $84.1 \pm 8.1 * * *$ & $77.1 \pm 12.4 * * *$ & $89.2 \pm 5.9 * * *$ \\
\hline & & 1 & $22.7 \pm 12.6$ & $38.7 \pm 4.7 * * *$ & $63.1 \pm 16.7 * * *$ & $81.7 \pm 9.9 * * *$ & $72.1 \pm 13.4 * * *$ & $80.5 \pm 9.9 * * *$ \\
\hline & & 0.5 & $6.6 \pm 3.5$ & $22.3 \pm 3.1 * * *$ & $47.3 \pm 18.4 *$ & $71.3 \pm 14.7 * * *$ & $59.4 \pm 15.0 * *$ & $67.8 \pm 10.5 * * *$ \\
\hline & & 0.2 & - & $7.0 \pm 0.7 *$ & $27.7 \pm 16.7$ & $64.2 \pm 15.4 * * *$ & $3.8 \pm 1.3$ & $20.9 \pm 6.8$ \\
\hline & & 0.1 & - & - & $4.8 \pm 3.1$ & $34.3 \pm 14.2 *$ & - & $12.8 \pm 4.1 *$ \\
\hline & & 0.05 & - & - & $2.1 \pm 2.3$ & $8.0 \pm 2.2$ & - & $1.8 \pm 0.8$ \\
\hline$I C_{50}(\mu \mathrm{g} / \mathrm{mL})$ & - & - & 2.72 & 1.04 & 0.72 & 0.23 & 0.65 & 0.45 \\
\hline $\operatorname{Col}(10 \mu \mathrm{M})$ & $0.0 \pm 0.8$ & 10 & $20.1 \pm 2.1 * * *$ & $28.3 \pm 6.3 * *$ & $41.6 \pm 4.7 * * *$ & - & $6.6 \pm 1.9 *$ & $49.3 \pm 6.3 * * *$ \\
\hline PAF $(2 \mathrm{ng} / \mathrm{mL})$ & $0.0 \pm 1.0$ & 10 & $-0.6 \pm 0.4$ & $0.4 \pm 0.0$ & $0.2 \pm 0.2$ & - & $-0.4 \pm 0.6$ & $-0.3 \pm 0.3$ \\
\hline $\operatorname{Thr}(0.1 \mathrm{U} / \mathrm{mL})$ & $0.0 \pm 1.4$ & 10 & $-0.5 \pm 0.2$ & $-2.7 \pm 1.2$ & $-1.2 \pm 0.3$ & $0.7 \pm 0.1$ & $-1.6 \pm 0.5$ & $-4.3 \pm 0.1$ \\
\hline
\end{tabular}

Table 11. Antiplatelet activity of $[n]$-dehydrogingerols $\mathbf{3 a}-\mathbf{f}$.

\begin{tabular}{|c|c|c|c|c|c|c|c|c|}
\hline \multicolumn{9}{|c|}{ Inhibition (\%) } \\
\hline Inducer & Control & Conc. $(\mu \mathrm{g} / \mathrm{mL})$ & 3a & $\mathbf{3 b}$ & $3 \mathrm{c}$ & 3d & $3 e$ & $3 f$ \\
\hline \multirow{5}{*}{ AA $(100 \mu \mathrm{M})$} & \multirow{5}{*}{$0.0 \pm 0.8$} & 10 & $78.0 \pm 12.7 * * *$ & $98.8 \pm 0.2 * * *$ & $100.0 \pm 0.8 * * *$ & $84.7 \pm 12.4 * * *$ & $92.9 \pm 5.0 * * *$ & $100.0 \pm 0.8 * * *$ \\
\hline & & 5 & $63.0 \pm 14.3 * * *$ & $53.7 \pm 11.1 * *$ & $76.2 \pm 12.0 * * *$ & $65.5 \pm 16.5 * * *$ & $83.2 \pm 12.9 * * *$ & $100.0 \pm 0.8 * * *$ \\
\hline & & 2 & $30.4 \pm 19.4$ & $25.7 \pm 13.8$ & $15.3 \pm 3.9 * * *$ & $52.4 \pm 21.7 *$ & $19.6 \pm 3.9 * * *$ & $100.0 \pm 0.8 * * *$ \\
\hline & & 1 & $8.5 \pm 6.9$ & $16.4 \pm 13.5$ & $7.0 \pm 3.5$ & $4.4 \pm 1.0 *$ & $5.7 \pm 0.2 * * *$ & $9.8 \pm 3.0 * *$ \\
\hline & & 0.5 & $2.8 \pm 1.7$ & $6.5 \pm 4.8$ & - & - & - & $2.6 \pm 0.9$ \\
\hline$I C_{50}(\mu \mathrm{g} / \mathrm{mL})$ & - & - & 3.59 & 4.74 & 3.19 & 2.99 & 3.14 & 1.20 \\
\hline $\mathrm{Col}(10 \mu \mathrm{M})$ & $0.0 \pm 0.6$ & 10 & $7.5 \pm 4.2$ & $16.9 \pm 8.4$ & $8.7 \pm 3.7$ & $10.6 \pm 3.3$ & $40.0 \pm 7.1 * * *$ & $41.3 \pm 14.2 *$ \\
\hline PAF (2 ng/mL) & $0.0 \pm 1.3$ & 10 & $3.1 \pm 1.0$ & $4.3 \pm 0.8$ & $1.4 \pm 0.2$ & $1.5 \pm 0.8$ & $3.0 \pm 1.8$ & $-0.9 \pm 0.6$ \\
\hline $\operatorname{Thr}(0.1 \mathrm{U} / \mathrm{mL})$ & $0.0 \pm 0.4$ & 10 & $1.9 \pm 0.3$ & $1.7 \pm 1.0$ & $0.7 \pm 0.6$ & $0.4 \pm 0.6$ & $1.0 \pm 0.4$ & $1.7 \pm 0.1$ \\
\hline
\end{tabular}


Table 12. Antiplatelet activity of $[n]$-isodehydrogingerdiones $\mathbf{1 a - c}$ and $\mathbf{e}$.

\begin{tabular}{|c|c|c|c|c|c|c|}
\hline \multicolumn{7}{|c|}{ Inhibition (\%) } \\
\hline Inducer & Control & Conc. $(\mu \mathrm{g} / \mathrm{mL})$ & $1 \mathbf{a}$ & $1 \mathbf{b}$ & $1 \mathrm{c}$ & $1 \mathrm{e}$ \\
\hline \multirow{6}{*}{ AA $(100 \mu \mathrm{M})$} & \multirow{6}{*}{$0.0 \pm 0.4$} & 10 & $100.0 \pm 0.4 * * *$ & $100.0 \pm 0.4 * * *$ & $98.4 \pm 0.9 * * *$ & $100.0 \pm 0.4 * * *$ \\
\hline & & 5 & $100.0 \pm 0.4 * * *$ & $100.0 \pm 0.4 * * *$ & - & $100.0 \pm 0.4 * * *$ \\
\hline & & 2 & $94.3 \pm 4.2 * * *$ & $93.1 \pm 5.5 * * *$ & $100.0 \pm 0.4 * * *$ & $82.7 \pm 6.0 * * *$ \\
\hline & & 1 & $30.8 \pm 6.4$ & $59.6 \pm 6.0 * * *$ & $53.4 \pm 4.2 * * *$ & $74.7 \pm 7.8 * * *$ \\
\hline & & 0.5 & $10.6 \pm 2.9 * *$ & $7.4 \pm 4.1$ & $40.3 \pm 9.9 * *$ & $26.0 \pm 5.4 * * *$ \\
\hline & & 0.2 & - & - & $3.6 \pm 1.6$ & $10.2 \pm 4.4$ \\
\hline$I C_{50}(\mu \mathrm{g} / \mathrm{mL})$ & - & - & 1.28 & 1.03 & 0.68 & 0.75 \\
\hline $\operatorname{Col}(10 \mu \mathrm{M})$ & $0.0 \pm 1.0$ & 10 & $53.3 \pm 6.3 * * *$ & $38.9 \pm 10.0 * * *$ & $35.2 \pm 6.6 * * *$ & $36.6 \pm 13.8 *$ \\
\hline PAF (2 ng/mL) & $0.0 \pm 1.0$ & 10 & $0.1 \pm 0.4$ & $0.2 \pm 0.4$ & $0.2 \pm 0.4$ & $2.3 \pm 0.3$ \\
\hline $\operatorname{Thr}(0.1 \mathrm{U} / \mathrm{mL})$ & $0.0 \pm 0.9$ & 10 & $1.7 \pm 0.2$ & $1.7 \pm 0.4$ & $0.2 \pm 0.1$ & $1.1 \pm 0.2$ \\
\hline
\end{tabular}

Table 13. Antiplatelet activity of $[n]$-epoxydehydroparadols $7 \mathbf{a}-\mathbf{f}$.

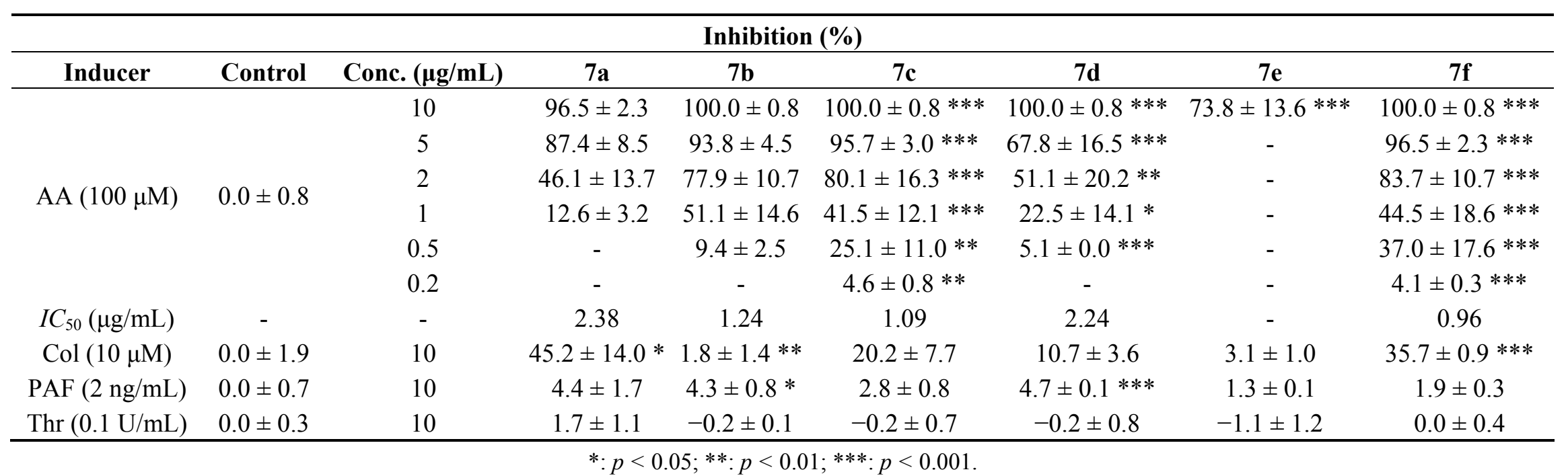


The addition of a $\beta$-hydroxyl group to the $\alpha, \beta$-unsaturated ketone to afford $[n]$-gingerols $\mathbf{8 a}-\mathbf{f}$, resulted in a reduction of anti-platelet aggregation activity (Table 10). The introduction of an unsaturated $\mathrm{C}=\mathrm{C}$ bond to the gingerol skeleton as described above (3a-f) also reduced the inhibition percentages (Table 11). Similarly, a longer side chain produced a more potent derivative. Therefore, both [10]-gingerol $\mathbf{8 f}$ and [10]-dehydrogingerol $\mathbf{3 f}$ displayed more significant inhibition of aggregation induced by AA $(100 \mu \mathrm{M})$ as compared to the analogues with shorter side chains.

The $[n]$-isodehydrogingerdiones 1a-e also showed significant inhibition of platelet aggregation induced by AA (Table 12). [7]-Isodehydrogingerdione 1c was found to be the most effective compound among this series, with an $I C_{50}$ value of $0.68 \mu \mathrm{g} / \mathrm{mL}$. Moreover, an epoxide ring next to the $\alpha, \beta$-unsaturated ketone produced derivatives $\mathbf{7 a}-\mathbf{f}$ of lower potency compared with $[n]$-paradols $\mathbf{5 a}-\mathbf{f}$. They were only as potent as the dehydroshogaol series, with $I C_{50}$ values between 0.96 and $2.38 \mu \mathrm{g} / \mathrm{mL}$. Apparently, [10]-epoxydehydroparadol $7 \mathbf{f}$ exhibited the most significant inhibitory effect among this series with an $I C_{50}$ value of $0.96 \mu \mathrm{g} / \mathrm{mL}$ (Table 13).

\section{Experimental Section}

\subsection{General}

All the chemicals were purchased from Merck KGaA (Darmstadt, Germany), unless specifically indicated. Column chromatography was performed on silica gel (70-230 mesh, 230-400 mesh), and TLC monitoring was executed on Merck precoated Si gel $60 \mathrm{~F}_{254}$ plates, using UV light to visualize the spots. The melting points of the purified compounds were determined using a Yanagimoto micromelting point measuring apparatus (Tokyo, Japan) without corrections. The UV spectra were obtained on a Hitachi UV-3210 spectrophotometer (Tokyo, Japan). The IR spectra were obtained as $\mathrm{KBr}$ discs on a Jasco Report-100 FT-IR spectrometer (Tokyo, Japan). ${ }^{1} \mathrm{H}$ and ${ }^{13} \mathrm{C}$ NMR spectra were recorded on a Bruker AC-200 NMR spectrometer (Bruker, Billerica, MA, USA). Chemical shifts are shown in $\delta$ values (ppm) with tetramethylsilane as an internal standard. The EI mass and high-resolution mass spectra were measured on a VG Analytical Model 70-250S spectrometer (Micromass, Manchester, UK). Elemental analyses were performed on a Perkin-Elmer 240 analyzer (Waltham, MA, USA).

\subsection{Synthesis of Derivatives and Spectral Data}

\subsubsection{Preparation of Dehydrozingerone (9)}

$10 \%$ Sodium hydroxide $(7.0 \mathrm{~g}, 175 \mathrm{mmol})$ was added dropwise to a solution of vanillin $(2.5 \mathrm{~g}$, $16.4 \mathrm{mmol})$ in acetone $(100 \mathrm{~mL})$ at room temperature. The reaction mixture was stirred for $12 \mathrm{~h}$, concentrated under reduced pressure, then neutralized by cold $5 \% \mathrm{HCl}_{(\mathrm{aq})}$. The solution was extracted with EtOAc $(4 \times 50 \mathrm{~mL})$. The organic layers were combined, washed with saturated $\mathrm{NaCl}_{(\mathrm{aq})}(\mathrm{brine})$, dried over $\mathrm{MgSO}_{4}$, and concentrated under reduced pressure. The product was isolated on silica gel column chromatography (EtOAc/hexanes $=1 / 4)$ to afford yellow needles ( $2.8 \mathrm{~g}, 89 \%$ yield).

Dehydrozingerone (9): $\mathrm{mp} 126-127{ }^{\circ} \mathrm{C}$ (lit. 128-129 ${ }^{\circ} \mathrm{C}$ ) [1]; UV (MeOH) $\lambda_{\max } 337,299$ (sh), $249 \mathrm{~nm}$; IR (KBr) $v_{\max } 3312,2949,2848,1670,1639,1581,1515,1427,1298,1218,1024,829 \mathrm{~cm}^{-1}$; ${ }^{1} \mathrm{H}-\mathrm{NMR}\left(\mathrm{CDCl}_{3}\right) \delta 7.44(1 \mathrm{H}, \mathrm{d}, J=16.0 \mathrm{~Hz}, \mathrm{H}-1), 7.09(1 \mathrm{H}, \mathrm{dd}, J=8.2,1.8 \mathrm{~Hz}, \mathrm{H}-6$ '), $7.05(1 \mathrm{H}, \mathrm{d}$, 
$\left.J=1.8 \mathrm{~Hz}, \mathrm{H}-2^{\prime}\right), 6.92(1 \mathrm{H}, \mathrm{d}, J=8.0 \mathrm{~Hz}, \mathrm{H}-5$ ') $, 6.58(1 \mathrm{H}, \mathrm{d}, J=16.0 \mathrm{~Hz}, \mathrm{H}-2), 6.02(1 \mathrm{H}, \mathrm{br} \mathrm{s},-\mathrm{OH})$, $3.93\left(3 \mathrm{H}, \mathrm{s},-\mathrm{OCH}_{3}\right), 2.36(3 \mathrm{H}, \mathrm{s}, \mathrm{H}-4) ;{ }^{13} \mathrm{C}-\mathrm{NMR}\left(\mathrm{CDCl}_{3}\right) \delta 198.4,148.2,146.7,143.7,126.8,124.9$, 123.4, 114.7, 109.2, 55.9, 27.2; EIMS m/z (rel. int.) $192\left(\mathrm{M}^{+}, 93\right), 190$ (20), 177 (100), 145 (47), 134 (21), 117 (27), 89 (31), 78 (23), 77 (24), 51 (24).

3.2.2. General Procedure for the Synthesis of $[n]$-Dehydroshogaols (2a-f) and $[n]$-Dehydrogingerols (3a-f)

A 1.0 M THF solution of lithium bis(trimethylsilyl)amide $(20.8 \mathrm{~mL})$ was added dropwise to a solution of dehydrozingerone (9) $(2.0 \mathrm{~g}, 10.4 \mathrm{mmol})$ in dry THF $(10 \mathrm{~mL})$ at $0{ }^{\circ} \mathrm{C}$ under argon. After the mixture had been stirred for $1 \mathrm{~h}$, the appropriate aldehyde $(10.5 \mathrm{mmol})$ was added and stirred for another $15 \mathrm{~min}$. The reaction was then quenched with $5 \% \mathrm{HCl}_{(\mathrm{aq})}$ at $0{ }^{\circ} \mathrm{C}$ and extracted with EtOAc $(4 \times 20 \mathrm{~mL})$. The organic layers were combined, washed with brine, dried over $\mathrm{MgSO}_{4}$, and concentrated under reduced pressure. Products $\mathbf{2}$ and $\mathbf{3}$ were isolated using silica gel column chromatography $\left(\mathrm{EtOAc} / \mathrm{CH}_{2} \mathrm{Cl}_{2}=1 / 16\right)$.

[5]-Dehydroshogaol (2a): yellow syrup (9\%); UV (MeOH) $\lambda_{\max }(\log \varepsilon) 356$ (4.14), 255 (4.03) nm; IR (neat) $v_{\max } 3325,2958,2860,1652,1625,1579,1514,1460,1276,1126,1031 \mathrm{~cm}^{-1} ;{ }^{1} \mathrm{H}-\mathrm{NMR}\left(\mathrm{CDCl}_{3}\right)$ $\delta 7.57(1 \mathrm{H}, \mathrm{d}, J=15.8 \mathrm{~Hz}, \mathrm{H}-1), 7.13(1 \mathrm{H}, \mathrm{dd}, J=8.2,2.0 \mathrm{~Hz}, \mathrm{H}-6$ '), 7.06 (1H, d, $J=2.0 \mathrm{~Hz}, \mathrm{H}-2$ '), $6.99(1 \mathrm{H}, \mathrm{dt}, J=15.6,6.8 \mathrm{~Hz}, \mathrm{H}-5), 6.92(1 \mathrm{H}, \mathrm{d}, J=8.2 \mathrm{~Hz}, \mathrm{H}-5$ '), 6.80 (1H, d, $J=15.8 \mathrm{~Hz}, \mathrm{H}-2)$, $6.44(1 \mathrm{H}, \mathrm{dt}, J=15.6,1.4 \mathrm{~Hz}, \mathrm{H}-4), 6.04(1 \mathrm{H}, \mathrm{br} \mathrm{s},-\mathrm{OH}), 3.93\left(3 \mathrm{H}, \mathrm{s},-\mathrm{OCH}_{3}\right), 2.28(2 \mathrm{H}, \mathrm{tdd}, J=6.8$, 6.8, $1.4 \mathrm{~Hz}, \mathrm{H}-6), 1.57-1.26$ (4H, m, H-7, -8), $0.92(3 \mathrm{H}, \mathrm{t}, J=7.0 \mathrm{~Hz}, \mathrm{H}-9) ;{ }^{13} \mathrm{C}-\mathrm{NMR}\left(\mathrm{CDCl}_{3}\right) \delta$ 189.3, 148.2, 148.0, 146.8, 143.3, 129.0, 127.4, 123.3, 122.8, 114.8, 109.7, 56.0, 32.4, 30.3, 22.3, 13.8; EIMS m/z (rel. int.) $260\left(\mathrm{M}^{+}, 66\right), 259$ (26), 217 (56), 177 (80), 168 (45), 152 (65), 151 (100), 137 (40), 123 (21), 111 (35), 97 (35), 91 (23), 71 (44), 69 (55), 57 (94), 55 (85); HREIMS m/z 260.1410 $[\mathrm{M}]^{+}$(Calcd for $\mathrm{C}_{16} \mathrm{H}_{20} \mathrm{O}_{3}, 260.1412$ ).

[5]-Dehydrogingerol (3a): yellow needles (65\%), mp $143-144{ }^{\circ} \mathrm{C}$ (lit. $144-146{ }^{\circ} \mathrm{C}$ ) [22]; UV $(\mathrm{MeOH}) \lambda_{\max }(\log \varepsilon) 340$ (4.46), 271 (sh) (3.62), 244 (4.11) nm; IR (KBr) $v_{\max }$ 3341, 3150, 2926, 2855, 1626, 1583, 1514, 1284, 1031, 972, $816 \mathrm{~cm}^{-1} ;{ }^{1} \mathrm{H}-\mathrm{NMR}\left(\mathrm{CDCl}_{3}\right) \delta 7.50(1 \mathrm{H}, \mathrm{d}, J=16.2 \mathrm{~Hz}, \mathrm{H}-1)$, $7.11\left(1 \mathrm{H}, \mathrm{dd}, J=8.0,1.8 \mathrm{~Hz}, \mathrm{H}-6\right.$ ') $7.05\left(1 \mathrm{H}, \mathrm{d}, J=1.8 \mathrm{~Hz}, \mathrm{H}-2^{\prime}\right), 6.93$ (1H, d, $J=8.0 \mathrm{~Hz}, \mathrm{H}-5$ '), $6.58(1 \mathrm{H}, \mathrm{d}, J=16.2 \mathrm{~Hz}, \mathrm{H}-2), 6.02(1 \mathrm{H}, \mathrm{br} \mathrm{s},-\mathrm{OH}), 4.13(1 \mathrm{H}, \mathrm{m}, \mathrm{H}-5), 3.93\left(3 \mathrm{H}, \mathrm{s},-\mathrm{OCH}_{3}\right), 2.88(1 \mathrm{H}$, $\mathrm{dd}, J=17.0,2.8 \mathrm{~Hz}, \mathrm{H}-4), 2.73(1 \mathrm{H}, \mathrm{dd}, J=17.0,8.8 \mathrm{~Hz}, \mathrm{H}-4), 1.58-1.35$ (6H, m, H-6 8), 0.92 (3H, $\mathrm{t}, J=6.6 \mathrm{~Hz}, \mathrm{H}-9) ;{ }^{13} \mathrm{C}-\mathrm{NMR}\left(\mathrm{CDCl}_{3}\right) \delta 200.9,148.5,146.8,143.8,126.6,124.1,123.7,114.8,109.4$, 67.9, 56.0, 46.4, 36.2, 27.7, 22.6, 14.0; EIMS m/z (rel. int.) $278\left(\mathrm{M}^{+}, 33\right), 192$ (37), 177 (100), 150 (38), 145 (37), 137 (38), 89 (14); Anal. Calcd for $\mathrm{C}_{16} \mathrm{H}_{20} \mathrm{O}_{4}$ : C, 69.06\%; H, 7.91\%; Found: C, 69.09\%; $\mathrm{H}, 7.85 \%$.

[6]-Dehydroshogaol (2b): yellow syrup (15\%); UV (MeOH) $\lambda_{\max }(\log \varepsilon) 355$ (4.02), 258 (3.93) nm; IR(neat) $v_{\max } 3354,2956,2856,1654,1625,1581,1514,1267,1207,1033 \mathrm{~cm}^{-1} ;{ }^{1} \mathrm{H}-\mathrm{NMR}\left(\mathrm{CDCl}_{3}\right)$ $\delta 7.58(1 \mathrm{H}, \mathrm{d}, J=15.8 \mathrm{~Hz}, \mathrm{H}-1), 7.14(1 \mathrm{H}, \mathrm{dd}, J=8.2,1.8 \mathrm{~Hz}, \mathrm{H}-6$ ') 7.07 (1H, d, $J=1.8 \mathrm{~Hz}, \mathrm{H}-2$ '), $7.00(1 \mathrm{H}, \mathrm{dt}, J=15.6,7.0 \mathrm{~Hz}, \mathrm{H}-5), 6.94(1 \mathrm{H}, \mathrm{d}, J=8.2 \mathrm{~Hz}, \mathrm{H}-5$ '), $6.81(1 \mathrm{H}, \mathrm{d}, J=15.8 \mathrm{~Hz}, \mathrm{H}-2)$, $6.43(1 \mathrm{H}, \mathrm{dt}, J=15.6,1.4 \mathrm{~Hz}, \mathrm{H}-4), 5.97(1 \mathrm{H}, \mathrm{br} \mathrm{s},-\mathrm{OH}), 3.94\left(3 \mathrm{H}, \mathrm{s},-\mathrm{OCH}_{3}\right), 2.27(2 \mathrm{H}, \mathrm{tdd}, J=7.0$, 6.8, $1.4 \mathrm{~Hz}, \mathrm{H}-6), 1.57-1.25$ (6H, m, H-7 9), 0.90 (3H, t, $J=6.7 \mathrm{~Hz}, \mathrm{H}-10) ;{ }^{13} \mathrm{C}-\mathrm{NMR}\left(\mathrm{CDCl}_{3}\right)$ 
$\delta 189.3,148.1,148.0,147.2,143.3,129.0,127.4,123.3,122.8,114.8,109.7,56.0,32.7,31.4,27.9$, 22.4, 14.0; EIMS m/z (rel. int.) $274\left(\mathrm{M}^{+}, 100\right), 273$ (36), 217 (82), 177 (81), 152 (21), 151 (36), 145 (20), 137 (45), 57 (36), 55 (44); HREIMS $m / z 274.1571[\mathrm{M}]^{+}$(Calcd for $\mathrm{C}_{17} \mathrm{H}_{22} \mathrm{O}_{3}, 274.1568$ ).

[6]-Dehydrogingerol (3b): yellow needles (59\%), mp 123-124 ${ }^{\circ} \mathrm{C}$ (lit. 134-136 ${ }^{\circ} \mathrm{C}$ ) [22]; UV (MeOH) $\lambda_{\max }(\log \varepsilon) 341$ (4.34), 270 (sh) (3.59), 247 (3.98) nm; IR (KBr) $v_{\max }$ 3460, 3161, 2962, 2858, 1675, 1589, 1517, 1433, 1281, 1223, 1174, 1076, $872 \mathrm{~cm}^{-1} ;{ }^{1} \mathrm{H}-\mathrm{NMR}\left(\mathrm{CDCl}_{3}\right) \delta 7.50(1 \mathrm{H}, \mathrm{d}, J=16.0 \mathrm{~Hz}$, H-1), $7.11(1 \mathrm{H}, \mathrm{dd}, J=8.0,2.0 \mathrm{~Hz}, \mathrm{H}-6$ ') $7.05(1 \mathrm{H}, \mathrm{d}, J=2.0 \mathrm{~Hz}, \mathrm{H}-2$ '), $6.93(1 \mathrm{H}, \mathrm{d}, J=8.2 \mathrm{~Hz}$, H-5'), $6.58(1 \mathrm{H}, \mathrm{d}, J=16.0 \mathrm{~Hz}, \mathrm{H}-2), 4.14(1 \mathrm{H}, \mathrm{m}, \mathrm{H}-5), 3.92\left(3 \mathrm{H}, \mathrm{s},-\mathrm{OCH}_{3}\right), 2.88(1 \mathrm{H}, \mathrm{dd}, J=17.2$, $3.2 \mathrm{~Hz}, \mathrm{H}-4), 2.72$ (1H, dd, $J=17.2,8.6 \mathrm{~Hz}, \mathrm{H}-4), 1.51-1.25$ (8H, m, H-6 9), 0.89 (3H, t, $J=6.4 \mathrm{~Hz}$, $\mathrm{H}-10) ;{ }^{13} \mathrm{C}-\mathrm{NMR}\left(\mathrm{CDCl}_{3}\right) \delta 200.1,150.2,148.7,143.8,127.6,125.1,124.1,116.1,111.4,68.5,56.2$, 48.4, 38.0, 32.6, 26.0, 23.3, 14.3; EIMS m/z (rel. int.) $292\left(\mathrm{M}^{+}, 51\right), 192$ (20), 177 (100), 150 (47), $137(40), 89(10)$.

[7]-Dehydroshogaol (2c): yellow syrup (13\%); UV (MeOH) $\lambda_{\max }(\log \varepsilon) 357$ (3.91), 261 (3.95) nm; IR (neat) $v_{\max } 3384,2954,2856,1654,1625,1583,1514,1274,1124,1033 \mathrm{~cm}^{-1} ;{ }^{1} \mathrm{H}-\mathrm{NMR}\left(\mathrm{CDCl}_{3}\right)$ $\delta 7.58(1 \mathrm{H}, \mathrm{d}, J=15.8 \mathrm{~Hz}, \mathrm{H}-1), 7.14(1 \mathrm{H}, \mathrm{dd}, J=8.1,1.8 \mathrm{~Hz}, \mathrm{H}-6$ '), 7.08 (1H, d, $J=1.8 \mathrm{~Hz}, \mathrm{H}-2$ '), $7.00(1 \mathrm{H}, \mathrm{dt}, J=15.6,6.9 \mathrm{~Hz}, \mathrm{H}-5), 6.93(1 \mathrm{H}, \mathrm{d}, J=8.1 \mathrm{~Hz}, \mathrm{H}-5$ '), $6.81(1 \mathrm{H}, \mathrm{d}, J=15.8 \mathrm{~Hz}, \mathrm{H}-2)$, $6.43(1 \mathrm{H}, \mathrm{dt}, J=15.6,1.4 \mathrm{~Hz}, \mathrm{H}-4), 5.92(1 \mathrm{H}, \mathrm{br} \mathrm{s},-\mathrm{OH}), 3.94\left(3 \mathrm{H}, \mathrm{s},-\mathrm{OCH}_{3}\right), 2.27(2 \mathrm{H}, \mathrm{tdd}, J=6.9$, 6.9, $1.4 \mathrm{~Hz}, \mathrm{H}-6), 1.54-1.25(8 \mathrm{H}, \mathrm{m}, \mathrm{H}-7 \sim 10), 0.89(3 \mathrm{H}, \mathrm{t}, J=6.7 \mathrm{~Hz}, \mathrm{H}-11) ;{ }^{13} \mathrm{C}-\mathrm{NMR}\left(\mathrm{CDCl}_{3}\right)$ $\delta 189.3,148.2,148.0,146.8,143.4,129.0,127.3,123.3,122.7,114.8,109.7,55.9,32.7,31.6,28.9$, 28.1, 22.5, 14.0; EIMS m/z (rel. int.) 288 (M+1 100), 287 (48), 217 (82), 204 (27), 177 (49), 137 (33); HREIMS $m / z 288.1725[\mathrm{M}]^{+}$(Calcd for $\mathrm{C}_{18} \mathrm{H}_{24} \mathrm{O}_{3}, 288.1725$ ).

[7]-Dehydrogingerol (3c): yellow needles (56\%), mp 108-109 ${ }^{\circ} \mathrm{C}$ (lit. 110-112 ${ }^{\circ} \mathrm{C}$ ) [22]; $\mathrm{UV}(\mathrm{MeOH})$ $\lambda_{\max }(\log \varepsilon) 340$ (4.63), 271 (sh) (4.23), 252 (4.39) nm; IR (KBr) $v_{\max }$ 3447, 3258, 2926, 2855, 1694, 1589, 1512, 1437, 1279, 1221, 1053, $812 \mathrm{~cm}^{-1}$; ${ }^{1} \mathrm{H}-\mathrm{NMR}\left(\mathrm{CDCl}_{3}\right) \delta 7.46(1 \mathrm{H}, \mathrm{d}, J=16.0 \mathrm{~Hz}, \mathrm{H}-1)$, 7.04 (1H, dd, $J=8.2,1.8 \mathrm{~Hz}, \mathrm{H}-6$ ') 7.00 (1H, d, $\left.J=1.8 \mathrm{~Hz}, \mathrm{H}-2^{\prime}\right), 6.88$ (1H, d, $J=8.0 \mathrm{~Hz}, \mathrm{H}-5$ '), $6.53(1 \mathrm{H}, \mathrm{d}, J=16.0 \mathrm{~Hz}, \mathrm{H}-2), 4.17-4.06(1 \mathrm{H}, \mathrm{m}, \mathrm{H}-5), 3.87\left(3 \mathrm{H}, \mathrm{s},-\mathrm{OCH}_{3}\right), 2.85(1 \mathrm{H}, \mathrm{dd}, J=17.0$, $3.2 \mathrm{~Hz}, \mathrm{H}-4), 2.71(1 \mathrm{H}, \mathrm{dd}, J=17.0,8.6 \mathrm{~Hz}, \mathrm{H}-4), 1.58-1.25(10 \mathrm{H}, \mathrm{m}, \mathrm{H}-6 \sim 10), 0.85$ (3H, t, $J=6.6 \mathrm{~Hz}$, $\mathrm{H}-11) ;{ }^{13} \mathrm{C}-\mathrm{NMR}\left(\mathrm{CDCl}_{3}\right) \delta 201.0,148.6,147.0,143.9,126.7,124.1,123.7,115.0,109.5,68.0,56.0$, 46.5, 36.6, 31.8, 29.3, 25.5, 22.6, 14.0; EIMS m/z (rel. int.) $306\left(\mathrm{M}^{+}, 26\right), 217$ (23), 192 (44), 177 (100), 150 (34), 145 (38), 137 (17), 89 (14).

[8]-Dehydroshogaol (2d): yellow syrup (15\%); UV (MeOH) $\lambda_{\max }(\log \varepsilon) 357$ (4.10), 258 (3.96) nm; IR(neat) $v_{\max } 3395,2925,2856,1660,1614,1581,1514,1278,1174,1033 \mathrm{~cm}^{-1} ;{ }^{1} \mathrm{H}-\mathrm{NMR}\left(\mathrm{CDCl}_{3}\right)$ $\delta 7.58(1 \mathrm{H}, \mathrm{d}, J=16.0 \mathrm{~Hz}, \mathrm{H}-1), 7.12(1 \mathrm{H}, \mathrm{dd}, J=8.2,2.0 \mathrm{~Hz}, \mathrm{H}-6$ ') 7.06 (1H, d, $J=2.0 \mathrm{~Hz}, \mathrm{H}-2$ '), $6.99(1 \mathrm{H}, \mathrm{dt}, J=15.6,6.9 \mathrm{~Hz}, \mathrm{H}-5), 6.93(1 \mathrm{H}, \mathrm{d}, J=8.2 \mathrm{~Hz}, \mathrm{H}-5$ '), $6.81(1 \mathrm{H}, \mathrm{d}, J=16.0 \mathrm{~Hz}, \mathrm{H}-2)$, $6.43(1 \mathrm{H}, \mathrm{dt}, J=15.6,1.4 \mathrm{~Hz}, \mathrm{H}-4), 6.21(1 \mathrm{H}, \mathrm{br} \mathrm{s},-\mathrm{OH}), 3.91\left(3 \mathrm{H}, \mathrm{s},-\mathrm{OCH}_{3}\right), 2.26(2 \mathrm{H}, \mathrm{tdd}, J=6.9$, $6.9,1.4 \mathrm{~Hz}, \mathrm{H}-6), 1.52-1.27(10 \mathrm{H}, \mathrm{m}, \mathrm{H}-7-11), 0.88(3 \mathrm{H}, \mathrm{t}, J=6.8 \mathrm{~Hz}, \mathrm{H}-12) ;{ }^{13} \mathrm{C}-\mathrm{NMR}\left(\mathrm{CDCl}_{3}\right)$ $\delta 189.3,148.2,148.0,146.8,143.3,129.0,127.3,123.2,122.7,114.8,109.7,55.9,32.7,31.7,29.1$, 29.0, 28.1, 22.6, 14.0; EIMS m/z (rel. int.) 302 ( $\left.\mathrm{M}^{+}, 81\right), 301$ (29), 217 (68), 177 (100), 150 (21), 137 (33), 55 (24); HREIMS m/z 302.1879 [M] (Calcd for $\mathrm{C}_{19} \mathrm{H}_{26} \mathrm{O}_{3}, 302.1881$ ). 
[8]-Dehydrogingerol (3d): yellow needles (66\%), mp 83-84 ${ }^{\circ} \mathrm{C}$ (lit. 88-90 ${ }^{\circ} \mathrm{C}$ ) [22]; UV (MeOH) $\lambda_{\max }$ $(\log \varepsilon) 340$ (4.57), 270 (sh) (4.25), 247 (4.38) nm; IR (KBr) $v_{\max }$ 3451, 3215, 2924, 2855, 1680, 1585, 1510, 1433, 1280, 1116, $854 \mathrm{~cm}^{-1}$; ${ }^{1} \mathrm{H}-\mathrm{NMR}\left(\mathrm{CDCl}_{3}\right) \delta 7.51(1 \mathrm{H}, \mathrm{d}, J=16.0 \mathrm{~Hz}, \mathrm{H}-1), 7.10(1 \mathrm{H}, \mathrm{dd}$, $J=8.2,1.8 \mathrm{~Hz}, \mathrm{H}-6$ '), 7.06 (1H, d, $\left.J=1.8 \mathrm{~Hz}, \mathrm{H}-2^{\prime}\right), 6.93$ (1H, d, $J=8.2 \mathrm{~Hz}, \mathrm{H}-5$ '), 6.59 (1H, d, $J=16.0 \mathrm{~Hz}, \mathrm{H}-2), 4.17-4.06(1 \mathrm{H}, \mathrm{m}, \mathrm{H}-5), 3.94\left(3 \mathrm{H}, \mathrm{s},-\mathrm{OCH}_{3}\right), 2.88(1 \mathrm{H}, \mathrm{dd}, J=17.2,3.1 \mathrm{~Hz}, \mathrm{H}-4)$, $2.72(1 \mathrm{H}, \mathrm{dd}, J=17.2,8.7 \mathrm{~Hz}, \mathrm{H}-4), 1.56-1.26(12 \mathrm{H}, \mathrm{m}, \mathrm{H}-6 \sim 11), 0.88$ (3H, t, $J=6.4 \mathrm{~Hz}, \mathrm{H}-12)$; ${ }^{13} \mathrm{C}-\mathrm{NMR}\left(\mathrm{CDCl}_{3}\right) \delta 200.2,150.2,148.8,143.9,127.6,125.0,124.2,116.1,111.5,68.6,56.2,48.4$, 38.1, 32.5, 30.3, 30.0, 26.3, 23.2, 14.3; EIMS m/z (rel. int.) $320\left(\mathrm{M}^{+}, 22\right), 192$ (53), 177 (100), 150 (28), 145 (31), 137 (30), 84 (37), 69 (28), 57 (40), 55(55); Anal. Calcd. for $\mathrm{C}_{19} \mathrm{H}_{28} \mathrm{O}_{4}: \mathrm{C}, 71.25 \%$; $\mathrm{H}$, $8.75 \%$; Found: C, $71.26 \%$; H, 8.79\%.

[9]-Dehydroshogaol (2e): yellow syrup (13\%); UV (MeOH) $\lambda_{\max }(\log \varepsilon) 355$ (4.02), 260 (4.03) nm; IR (neat) $v_{\max } 3358,2925,2856,1641,1587,1525,1274,1120,1037 \mathrm{~cm}^{-1} ;{ }^{1} \mathrm{H}-\mathrm{NMR}\left(\mathrm{CDCl}_{3}\right) \delta 7.57(1 \mathrm{H}$, $\mathrm{d}, J=15.8 \mathrm{~Hz}, \mathrm{H}-1), 7.11(1 \mathrm{H}, \mathrm{dd}, J=8.2,1.8 \mathrm{~Hz}, \mathrm{H}-6$ '), 7.06 (1H, d, $J=1.8 \mathrm{~Hz}, \mathrm{H}-2$ '), 6.99 (1H, dt, $J=15.4,7.2 \mathrm{~Hz}, \mathrm{H}-5), 6.91(1 \mathrm{H}, \mathrm{d}, J=8.2 \mathrm{~Hz}, \mathrm{H}-5$ '), $6.80(1 \mathrm{H}, \mathrm{d}, J=15.8 \mathrm{~Hz}, \mathrm{H}-2), 6.42(1 \mathrm{H}, \mathrm{dt}$, $J=15.4,1.3 \mathrm{~Hz}, \mathrm{H}-4), 3.90\left(3 \mathrm{H}, \mathrm{s},-\mathrm{OCH}_{3}\right), 2.25(2 \mathrm{H}, \mathrm{tdd}, J=7.2,6.8,1.3 \mathrm{~Hz}, \mathrm{H}-6), 1.41-1.26(12 \mathrm{H}$, m, H-7 12), $0.86(3 \mathrm{H}, \mathrm{t}, J=6.6 \mathrm{~Hz}, \mathrm{H}-13) ;{ }^{13} \mathrm{C}-\mathrm{NMR}\left(\mathrm{CDCl}_{3}\right) \delta 189.4,148.3,148.1,146.9,143.4$, 129.0, 127.3, 123.3, 122.7, 114.9, 109.8, 56.0, 32.7, 31.8, 29.3, 29.2, 29.1, 28.2, 22.6, 14.1; EIMS $\mathrm{m} / \mathrm{z}$ (rel. int.) $316\left(\mathrm{M}^{+}, 100\right), 315$ (36), 217 (83), 204 (23), 177 (86), 137 (44), 55 (21); HREIMS m/z $316.2040[\mathrm{M}]^{+}\left(\right.$Calcd for $\left.\mathrm{C}_{20} \mathrm{H}_{28} \mathrm{O}_{3}, 316.2038\right)$.

[9]-Dehydrogingerol (3e): yellow needles (58\%), mp 93-94 ${ }^{\circ} \mathrm{C}$ (lit. 93-94 ${ }^{\circ} \mathrm{C}$ ) [22]; $\mathrm{UV}$ (MeOH) $\lambda_{\max }$ $(\log \varepsilon) 339$ (4.50), 270 (sh) (4.10), 250 (4.27) nm; IR (KBr) $v_{\max }$ 3451, 2926, 2854, 1676, 1583, 1516, 1460, 1280, 1170, 1031, 977, $810 \mathrm{~cm}^{-1} ;{ }^{1} \mathrm{H}-\mathrm{NMR}\left(\mathrm{CDCl}_{3}\right) \delta 7.50(1 \mathrm{H}, \mathrm{d}, J=16.0 \mathrm{~Hz}, \mathrm{H}-1), 7.10(1 \mathrm{H}$, dd, $J=8.2,1.8 \mathrm{~Hz}, \mathrm{H}-6$ '), 7.05 (1H, d, $J=1.8 \mathrm{~Hz}, \mathrm{H}-2$ '), 6.93 (1H, d, $J=8.2 \mathrm{~Hz}, \mathrm{H}-5$ '), 6.59 (1H, d, $J=16.0 \mathrm{~Hz}, \mathrm{H}-2), 4.17-4.06(1 \mathrm{H}, \mathrm{m}, \mathrm{H}-5), 3.94\left(3 \mathrm{H}, \mathrm{s},-\mathrm{OCH}_{3}\right), 2.87(1 \mathrm{H}, \mathrm{dd}, J=17.1,3.0 \mathrm{~Hz}, \mathrm{H}-4)$, $2.72(1 \mathrm{H}, \mathrm{dd}, J=17.1,8.7 \mathrm{~Hz}, \mathrm{H}-4), 1.56-1.28$ (14H, m, H-6 12), 0.88 (3H, t, $J=6.4 \mathrm{~Hz}, \mathrm{H}-13)$; ${ }^{13} \mathrm{C}-\mathrm{NMR}\left(\mathrm{CDCl}_{3}\right) \delta 200.1,150.1,148.7,143.8,127.6,125.0,124.1,116.1,111.4,68.5,56.2,48.4$, 38.0, 32.6, 30.4, 30.3, 30.0, 26.3, 23.2, 14.3; EIMS m/z (rel. int.) 334 (M+, 33), 316 (27), 217 (22), 192 (50), 177 (100), 150 (30), 145 (27), 137 (46), 57 (20).

[10]-Dehydroshogaol (2f): yellow syrup (6\%); UV (MeOH) $\lambda_{\max }(\log \varepsilon) 355$ (4.18), 257 (4.04) nm; IR (neat) $v_{\max } 3533,2925,2856,1660,1614,1581,1514,1278,1201,1120,1031 \mathrm{~cm}^{-1} ;{ }^{1} \mathrm{H}-\mathrm{NMR}\left(\mathrm{CDCl}_{3}\right)$ $\delta 7.58(1 \mathrm{H}, \mathrm{d}, J=15.9 \mathrm{~Hz}, \mathrm{H}-1), 7.13(1 \mathrm{H}, \mathrm{dd}, J=8.2,1.8 \mathrm{~Hz}, \mathrm{H}-6$ '), 7.06 (1H, d, $J=1.8 \mathrm{~Hz}, \mathrm{H}-2$ '), $6.99(1 \mathrm{H}, \mathrm{dt}, J=15.6,6.8 \mathrm{~Hz}, \mathrm{H}-5), 6.91(1 \mathrm{H}, \mathrm{d}, J=8.2 \mathrm{~Hz}, \mathrm{H}-5$ '), $6.81(1 \mathrm{H}, \mathrm{d}, J=15.9 \mathrm{~Hz}, \mathrm{H}-2)$, $6.42(1 \mathrm{H}, \mathrm{dt}, J=15.6,1.4 \mathrm{~Hz}, \mathrm{H}-4), 6.17(1 \mathrm{H}, \mathrm{br} \mathrm{s},-\mathrm{OH}), 3.92\left(3 \mathrm{H}, \mathrm{s},-\mathrm{OCH}_{3}\right), 2.25(2 \mathrm{H}, \mathrm{tdd}, J=6.8$, 6.8, $1.4 \mathrm{~Hz}, \mathrm{H}-6), 1.52-1.26(14 \mathrm{H}, \mathrm{m}, \mathrm{H}-7 \sim 13), 0.87(3 \mathrm{H}, \mathrm{t}, J=6.7 \mathrm{~Hz}, \mathrm{H}-14) ;{ }^{13} \mathrm{C}-\mathrm{NMR}\left(\mathrm{CDCl}_{3}\right) \delta$ $189.3,148.2,148.0,146.8,143.4,129.0,127.3,123.3,122.7,114.8,109.7,55.9,32.7,31.8,29.5$, 29.4, 29.3, 29.2, 28.2, 22.7, 14.1; EIMS m/z (rel. int.) $330\left(\mathrm{M}^{+}, 47\right), 217$ (37), 177 (100), 152 (53), 150

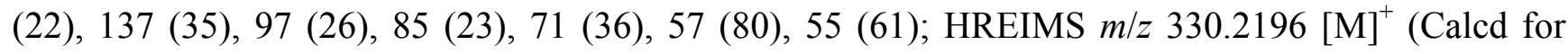
$\left.\mathrm{C}_{21} \mathrm{H}_{30} \mathrm{O}_{3}, 330.2194\right)$. 
[10]-Dehydrogingerol (3f): yellow needles (50\%), mp 74-75 ${ }^{\circ} \mathrm{C}$ (lit. 76-77.5 $\left.{ }^{\circ} \mathrm{C}\right)$ [22]; $\mathrm{UV}(\mathrm{MeOH})$ $\lambda_{\max }(\log \varepsilon) 340$ (4.27), 273 (sh) (3.68), 239 (4.06) nm; IR (KBr) $v_{\max }$ 3414, 2926, 2855, 1656, 1587, 1515, 1460, 1281, 1169, 1031, 979, $810 \mathrm{~cm}^{-1} ;{ }^{1} \mathrm{H}-\mathrm{NMR}\left(\mathrm{CDCl}_{3}\right) \delta 7.51(1 \mathrm{H}, \mathrm{d}, J=16.1 \mathrm{~Hz}, \mathrm{H}-1)$, $7.11\left(1 \mathrm{H}, \mathrm{dd}, J=8.1,1.8 \mathrm{~Hz}, \mathrm{H}-6\right.$ '), $7.05\left(1 \mathrm{H}, \mathrm{d}, J=1.8 \mathrm{~Hz}, \mathrm{H}-2^{\prime}\right), 6.93$ (1H, d, $J=8.1 \mathrm{~Hz}, \mathrm{H}-5$ '), $6.59(1 \mathrm{H}, \mathrm{d}, J=16.1 \mathrm{~Hz}, \mathrm{H}-2), 5.97(1 \mathrm{H}$, br s, $-\mathrm{OH}), 4.17-4.06(1 \mathrm{H}, \mathrm{m}, \mathrm{H}-5), 3.93\left(3 \mathrm{H}, \mathrm{s},-\mathrm{OCH}_{3}\right)$, $2.88(1 \mathrm{H}, \mathrm{dd}, J=17.1,3.0 \mathrm{~Hz}, \mathrm{H}-4), 2.73(1 \mathrm{H}, \mathrm{dd}, J=17.1,8.7 \mathrm{~Hz}, \mathrm{H}-4), 1.57-1.27(16 \mathrm{H}, \mathrm{m}$, $\mathrm{H}-6 \sim 13), 0.88(3 \mathrm{H}, \mathrm{t}, J=6.7 \mathrm{~Hz}, \mathrm{H}-14) ;{ }^{13} \mathrm{C}-\mathrm{NMR}\left(\mathrm{CDCl}_{3}\right) \delta 200.9,148.4,146.8,143.8,126.7,124.1$, 123.7, 114.8, 109.4, 68.0, 55.9, 46.5, 36.5, 31.8, $29.5(\times 2), 29.4,29.3,25.5,22.6,14.1$; EIMS $\mathrm{m} / \mathrm{z}$ (rel. int.) $348\left(\mathrm{M}^{+}, 24\right), 232$ (21), 192 (17), 177 (52), 150 (76), 145 (12), 137 (29), 97 (29), 91 (45), $57(100)$.

\subsubsection{General Procedure for the Synthesis of $[n]$-Epoxydehydroparadol $(\mathbf{7 a}-\mathbf{f})$}

A 1.0 M THF solution of lithium bis(trimethylsilyl)amide $(20.8 \mathrm{~mL})$ was added dropwise to a solution of dehydrozingerone (9) $(2.0 \mathrm{~g}, 10.4 \mathrm{mmol})$ in dry THF $(10 \mathrm{~mL})$ at $0{ }^{\circ} \mathrm{C}$ in an air atmosphere. After the mixture had been stirred for $1 \mathrm{~h}$, the appropriate aldehyde $(31.4 \mathrm{mmol})$ was added and stirred for $3 \mathrm{~h}$. The reaction was then quenched with $5 \% \mathrm{HCl}_{(\mathrm{aq})}$ at $0{ }^{\circ} \mathrm{C}$ and extracted with EtOAc $(4 \times 20 \mathrm{~mL})$. The organic layers were combined, washed with brine, dried over $\mathrm{Na}_{2} \mathrm{SO}_{4}$, and concentrated under reduced pressure. Products 2 and 7 were isolated using C-18 gel column chromatography (water/methanol $=1 / 2$ ).

1-(4-Hydroxy-3-methoxyphenyl)-4,5-expoxynon-1-en-3-one (7a): yellow syrup (8\%); UV (MeOH) $\lambda_{\max }(\log \varepsilon) 349$ (4.09), 251 (3.75) nm; IR (KBr) $v_{\max }$ 3451, 2956, 2931, 1693, 1587, 1514, 1465, 1271, $1031 \mathrm{~cm}^{-1}$; ${ }^{1} \mathrm{H}-\mathrm{NMR}\left(\mathrm{CDCl}_{3}\right) \delta 7.71(1 \mathrm{H}, \mathrm{d}, J=16.0 \mathrm{~Hz}, \mathrm{H}-1), 7.13(1 \mathrm{H}, \mathrm{dd}, J=8.2,1.8 \mathrm{~Hz}, \mathrm{H}-6$ '), $7.06\left(1 \mathrm{H}, \mathrm{d}, J=1.8 \mathrm{~Hz}, \mathrm{H}-2^{\prime}\right), 6.92(1 \mathrm{H}, \mathrm{d}, J=8.2 \mathrm{~Hz}, \mathrm{H}-5$ ') $6.71(1 \mathrm{H}, \mathrm{d}, J=16.0 \mathrm{~Hz}, \mathrm{H}-2), 6.01$ $(1 \mathrm{H}$, br s, $-\mathrm{OH}), 3.92\left(3 \mathrm{H}, \mathrm{s},-\mathrm{OCH}_{3}\right), 3.41(1 \mathrm{H}, \mathrm{d}, J=2.0 \mathrm{~Hz}, \mathrm{H}-4), 3.11(1 \mathrm{H}, \mathrm{td}, J=5.3,2.0 \mathrm{~Hz}$, $\mathrm{H}-5), 1.74-1.25$ (6H, m, H-6 8), $0.92(3 \mathrm{H}, \mathrm{t}, J=6.8 \mathrm{~Hz}, \mathrm{H}-9) ;{ }^{13} \mathrm{C}-\mathrm{NMR}\left(\mathrm{CDCl}_{3}\right) \delta 195.7,148.7$, 146.8, 145.2, 126.8, 124.2, 116.8, 114.8, 109.7, 59.6, 58.4, 56.0, 31.6, 27.9, 22.4, 13.9; EIMS m/z (rel. int.) $276\left(\mathrm{M}^{+}, 38\right), 177$ (100), 145 (20); HREIMS m/z 276.1363 [M] $]^{+}$(Calcd for $\left.\mathrm{C}_{16} \mathrm{H}_{20} \mathrm{O}_{4}, 276.1361\right)$.

1-(4-Hydroxy-3-methoxyphenyl)-4,5-expoxydec-1-en-3-one (7b): yellow syrup (10\%); UV (MeOH) $\lambda_{\max }(\log \varepsilon) 354(4.27), 251(3.93) \mathrm{nm}$; IR (neat) $v_{\max } 3414,2954,2862,1676,1585,1512,1460,1272$, $1031 \mathrm{~cm}^{-1}$; ${ }^{1} \mathrm{H}-\mathrm{NMR}\left(\mathrm{CDCl}_{3}\right) \delta 7.71(1 \mathrm{H}, \mathrm{d}, J=15.9 \mathrm{~Hz}, \mathrm{H}-1), 7.13(1 \mathrm{H}, \mathrm{dd}, J=8.1,1.8 \mathrm{~Hz}, \mathrm{H}-6$ '), $7.06(1 \mathrm{H}, \mathrm{d}, J=1.8 \mathrm{~Hz}, \mathrm{H}-2$ ') $6.91(1 \mathrm{H}, \mathrm{d}, J=8.1 \mathrm{~Hz}, \mathrm{H}-5$ ') $6.71(1 \mathrm{H}, \mathrm{d}, J=15.9 \mathrm{~Hz}, \mathrm{H}-2)$, $3.92\left(3 \mathrm{H}, \mathrm{s},-\mathrm{OCH}_{3}\right), 3.41(1 \mathrm{H}, \mathrm{d}, J=2.0 \mathrm{~Hz}, \mathrm{H}-4), 3.12(1 \mathrm{H}, \mathrm{td}, J=5.2,2.0 \mathrm{~Hz}, \mathrm{H}-5), 1.73-1.24$ $(8 \mathrm{H}, \mathrm{m}, \mathrm{H}-6 \sim 9), 0.89$ (3H, t, $J=6.8 \mathrm{~Hz}, \mathrm{H}-10) ;{ }^{13} \mathrm{C}-\mathrm{NMR}\left(\mathrm{CDCl}_{3}\right) \delta 195.6,148.6,146.7,145.2,126.8$, 124.2, 116.8, 114.8, 109.7, 59.5, 58.4, 56.0, 31.8, 31.4, 25.4, 22.4, 13.9; EIMS m/z (rel. int.) 290 (M $\left.\mathrm{M}^{+}, 39\right)$, 178 (21), 177 (100), 145 (22); HREIMS $m / z 290.1516$ [M] $]^{+}$(Calcd for $\mathrm{C}_{17} \mathrm{H}_{22} \mathrm{O}_{4}, 290.1518$ ).

1-(4-Hydroxy-3-methoxyphenyl)-4,5-expoxyundec-1-en-3-one (7c): yellow syrup (9\%); UV $(\mathrm{MeOH}) \lambda_{\max }(\log \varepsilon) 352$ (4.19), 254 (3.89) nm; IR (neat) $v_{\max }$ 3408, 2927, 2858, 1672, 1585, 1514, 1434, 1276, $1031 \mathrm{~cm}^{-1}$; ${ }^{1} \mathrm{H}-\mathrm{NMR}\left(\mathrm{CDCl}_{3}\right) \delta 7.71(1 \mathrm{H}, \mathrm{d}, J=15.8 \mathrm{~Hz}, \mathrm{H}-1), 7.13(1 \mathrm{H}, \mathrm{dd}, J=8.4$, $2.0 \mathrm{~Hz}, \mathrm{H}-6$ ') 7.06 (1H, d, $J=2.0 \mathrm{~Hz}, \mathrm{H}-2$ ') 6.91 (1H, d, $J=8.4 \mathrm{~Hz}, \mathrm{H}-5$ '), 6.70 (1H, d, $J=15.8 \mathrm{~Hz}$, 
$\mathrm{H}-2), 6.05(1 \mathrm{H}, \mathrm{br} \mathrm{s},-\mathrm{OH}), 3.92\left(3 \mathrm{H}, \mathrm{s},-\mathrm{OCH}_{3}\right), 3.41(1 \mathrm{H}, \mathrm{d}, J=2.0 \mathrm{~Hz}, \mathrm{H}-4), 3.13(1 \mathrm{H}, \mathrm{td}, J=5.0$, $2.0 \mathrm{~Hz}, \mathrm{H}-5), 1.73-1.29$ (10H, m, H-6 10), 0.88 (3H, t, $J=6.8 \mathrm{~Hz}, \mathrm{H}-11) ;{ }^{13} \mathrm{C}-\mathrm{NMR}\left(\mathrm{CDCl}_{3}\right) \delta 195.6$, 148.6, 146.7, 145.2, 126.8, 124.2, 116.8, 114.8, 109.7, 59.5, 58.4, 56.0, 31.8, 31.6, 28.9, 25.7, 22.4, 13.9 ; EIMS m/z (rel. int.) $304\left(\mathrm{M}^{+}, 46\right), 178$ (26), 177 (100), 145 (26); HREIMS m/z 304.1673 [M] ${ }^{+}$ (Calcd for $\mathrm{C}_{18} \mathrm{H}_{24} \mathrm{O}_{4}, 304.1674$ ).

1-(4-Hydroxy-3-methoxyphenyl)-4,5-expoxydodec-1-en-3-one (7d): yellow syrup (9\%); UV (MeOH) $\lambda_{\max }(\log \varepsilon) 351$ (4.33), 255 (4.07) nm; IR(neat) $v_{\max } 3395,2925,2858,1672,1581,1514,1434,1172$, $1031 \mathrm{~cm}^{-1} ;{ }^{1} \mathrm{H}-\mathrm{NMR}\left(\mathrm{CDCl}_{3}\right) \delta 7.70(1 \mathrm{H}, \mathrm{d}, J=16.0 \mathrm{~Hz}, \mathrm{H}-1), 7.12(1 \mathrm{H}, \mathrm{dd}, J=8.2,2.0 \mathrm{~Hz}, \mathrm{H}-6$ '), $7.05(1 \mathrm{H}, \mathrm{d}, J=2.0 \mathrm{~Hz}, \mathrm{H}-2$ ') $6.90(1 \mathrm{H}, \mathrm{d}, J=8.2 \mathrm{~Hz}, \mathrm{H}-5$ ') $, 6.70(1 \mathrm{H}, \mathrm{d}, J=16.0 \mathrm{~Hz}, \mathrm{H}-2), 6.16$ $(1 \mathrm{H}$, br s, $-\mathrm{OH}), 3.91\left(3 \mathrm{H}, \mathrm{s},-\mathrm{OCH}_{3}\right), 3.41(1 \mathrm{H}, \mathrm{d}, J=2.0 \mathrm{~Hz}, \mathrm{H}-4), 3.12(1 \mathrm{H}, \mathrm{td}, J=5.2,2.0 \mathrm{~Hz}$, H-5), 1.73-1.26 (12H, m, H-6 11), 0.87 (3H, t, $J=6.8 \mathrm{~Hz}, \mathrm{H}-12) ;{ }^{13} \mathrm{C}-\mathrm{NMR}\left(\mathrm{CDCl}_{3}\right) \delta$ 195.7, 148.7, 146.8, 145.2, 126.8, 124.2, 116.8, 114.8, 109.7, 59.5, 58.4, 56.0, 31.8, 31.7, 29.2, 29.1, 25.8, 22.6, 14.0; EIMS m/z (rel. int.) $318\left(\mathrm{M}^{+}, 37\right), 178$ (22), 177 (100), 145 (19); HREIMS m/z 318.1834 [M] $]^{+}$ (Calcd for $\mathrm{C}_{19} \mathrm{H}_{26} \mathrm{O}_{4}, 318.1831$ ).

1-(4-Hydroxy-3-methoxyphenyl)-4,5-expoxytridec-1-en-3-one (7e): yellow syrup (8\%); UV (MeOH) $\lambda_{\max }(\log \varepsilon) 353$ (4.32), $254(4.04) \mathrm{nm}$; IR (neat) $v_{\max }$ 3404, 2925, 2856, 1672, 1583, 1514, 1434, 1276, $1031 \mathrm{~cm}^{-1} ;{ }^{1} \mathrm{H}-\mathrm{NMR}\left(\mathrm{CDCl}_{3}\right) \delta 7.72(1 \mathrm{H}, \mathrm{d}, J=15.8 \mathrm{~Hz}, \mathrm{H}-1), 7.14(1 \mathrm{H}, \mathrm{dd}, J=8.2,1.8 \mathrm{~Hz}, \mathrm{H}-6$ '), $7.07\left(1 \mathrm{H}, \mathrm{d}, J=1.8 \mathrm{~Hz}, \mathrm{H}-2^{\prime}\right), 6.92(1 \mathrm{H}, \mathrm{d}, J=8.2 \mathrm{~Hz}, \mathrm{H}-5$ ') $, 6.71(1 \mathrm{H}, \mathrm{d}, J=15.8 \mathrm{~Hz}, \mathrm{H}-2), 5.95$ $(1 \mathrm{H}$, br s, $-\mathrm{OH}), 3.94\left(3 \mathrm{H}, \mathrm{s},-\mathrm{OCH}_{3}\right), 3.41(1 \mathrm{H}, \mathrm{d}, J=2.0 \mathrm{~Hz}, \mathrm{H}-4), 3.13(1 \mathrm{H}, \mathrm{td}, J=5.0,2.0 \mathrm{~Hz}$, H-5), 1.74-1.27 (14H, m, H-6 12), $0.88(3 \mathrm{H}, \mathrm{t}, J=6.8 \mathrm{~Hz}, \mathrm{H}-13) ;{ }^{13} \mathrm{C}-\mathrm{NMR}\left(\mathrm{CDCl}_{3}\right) \delta$ 195.6, 148.7, 146.8, 145.1, 126.9, 124.2, 116.9, 114.8, 109.7, 59.6, 58.4, 56.0, 31.8 (×2), 29.4, 29.3, 29.1, 25.8, 22.6, 14.0; EIMS m/z (rel. int.) $332\left(\mathrm{M}^{+}, 36\right), 177$ (100), 145 (15), 55 (12); HREIMS m/z 332.1990 [M] (Calcd for $\mathrm{C}_{20} \mathrm{H}_{28} \mathrm{O}_{4}, 332.1987$ ).

1-(4-Hydroxy-3-methoxyphenyl)-4,5-expoxytetradec-1-en-3-one (7f): yellow syrup (8\%); UV $(\mathrm{MeOH}) \lambda_{\max }(\log \varepsilon) 350$ (4.19), $253(4.17) \mathrm{nm}$; IR (neat) $v_{\max }$ 3423, 2925, 2854, 1676, 1585, 1512, 1460, 1274, $1031 \mathrm{~cm}^{-1} ;{ }^{1} \mathrm{H}-\mathrm{NMR}\left(\mathrm{CDCl}_{3}\right) \delta 7.71(1 \mathrm{H}, \mathrm{d}, J=15.8 \mathrm{~Hz}, \mathrm{H}-1), 7.14(1 \mathrm{H}, \mathrm{dd}, J=8.2$, $1.8 \mathrm{~Hz}, \mathrm{H}-6$ ') 7.07 (1H, d, $J=1.8 \mathrm{~Hz}, \mathrm{H}-2$ '), 6.92 (1H, d, $J=8.2 \mathrm{~Hz}, \mathrm{H}-5$ '), $6.72(1 \mathrm{H}, \mathrm{d}, J=15.8 \mathrm{~Hz}$, $\mathrm{H}-2), 5.90(1 \mathrm{H}$, br s, $-\mathrm{OH}), 3.94\left(3 \mathrm{H}, \mathrm{s},-\mathrm{OCH}_{3}\right), 3.41(1 \mathrm{H}, \mathrm{d}, J=2.0 \mathrm{~Hz}, \mathrm{H}-4), 3.13(1 \mathrm{H}, \mathrm{td}, J=5.4$, $2.0 \mathrm{~Hz}, \mathrm{H}-5), 1.74-1.27$ (16H, m, H-6 13), 0.88 (3H, t, $J=6.8 \mathrm{~Hz}, \mathrm{H}-14) ;{ }^{13} \mathrm{C}-\mathrm{NMR}\left(\mathrm{CDCl}_{3}\right) \delta 195.7$, 148.7, 146.8, 145.2, 126.9, 124.2, 116.8, 114.8, 109.7, 59.6, 58.4, 56.0, 31.9, 31.8, 29.5, 29.4, 29.3, 29.2, 25.8, 22.6, 14.1; EIMS m/z (rel. int.) $346\left(\mathrm{M}^{+}, 33\right), 177$ (100), 151 (23), 150 (55), 55 (20); HREIMS $m / z$ 346.2145 [M] (Calcd for $\mathrm{C}_{21} \mathrm{H}_{30} \mathrm{O}_{4}, 346.2144$ ).

\subsubsection{General Procedure for the Synthesis of $[n]$-Paradols $(\mathbf{5 a}-\mathbf{f})$}

A solution of $[n]$-dehydroshogaols $(\mathbf{2 a}-\mathbf{f})(0.96 \mathrm{mmol})$ in ethyl acetate $(20 \mathrm{~mL})$ containing palladium-charcoal $(5 \%, 0.05 \mathrm{~g})$ was stirred under hydrogen at atmospheric pressure and room temperature for $30 \mathrm{~min}$. The reaction mixture was monitored by TLC until no starting material remained. The catalyst was removed through celite, and the filtrate was concentrated under reduced pressure. The product was isolated using silica gel column chromatography $($ EtOAc/hexanes $=1 / 4)$. 
[5]-Paradol (5a): colorless syrup (79\%) [23]; UV (MeOH) $\lambda_{\max }(\log \varepsilon) 282$ (3.41) nm; IR (neat) $v_{\max }$ 3439, 2939, 2862, 1707, 1608, 1516, 1452, 1365, 1269, 1031, $806 \mathrm{~cm}^{-1} ;{ }^{1} \mathrm{H}-\mathrm{NMR}\left(\mathrm{CDCl}_{3}\right) \delta 6.79$ $(1 \mathrm{H}, \mathrm{d}, J=8.0 \mathrm{~Hz}, \mathrm{H}-5$ ') $6.67(1 \mathrm{H}, \mathrm{d}, J=1.8 \mathrm{~Hz}, \mathrm{H}-2$ '), $6.63(1 \mathrm{H}, \mathrm{dd}, J=8.0,1.8 \mathrm{~Hz}, \mathrm{H}-6$ '), $3.82\left(3 \mathrm{H}, \mathrm{s},-\mathrm{OCH}_{3}\right), 2.84-2.63(4 \mathrm{H}, \mathrm{m}, \mathrm{H}-1,-2), 2.35(2 \mathrm{H}, \mathrm{t}, J=7.2 \mathrm{~Hz}, \mathrm{H}-4), 1.58-1.51(2 \mathrm{H}, \mathrm{m}$, $\mathrm{H}-5), 1.23$ (6H, m, H-6 8), 0.87 (3H, t, $J=6.2 \mathrm{~Hz}, \mathrm{H}-9) ;{ }^{13} \mathrm{C}-\mathrm{NMR}\left(\mathrm{CDCl}_{3}\right) \delta 210.8,146.4,143.8$, 132.9, 120.6, 114.3, 111.1, 55.7, 44.5, 43.0, 31.5, 29.4, 28.8, 23.7, 22.4, 14.0; EIMS m/z (rel. int.) 264 $\left(\mathrm{M}^{+}, 58\right), 179$ (19), 151 (22), 137 (100); HREIMS m/z 246.1729 [M] (Calcd for $\left.\mathrm{C}_{16} \mathrm{H}_{24} \mathrm{O}_{3}, 246.1725\right)$.

[6]-Paradol (5b): colorless syrup (78\%) [23]; UV (MeOH) $\lambda_{\max }(\log \varepsilon) 281$ (3.34) nm; IR (neat) $v_{\max }$ 3451, 2940, 2862, 1713, 1516, 1452, 1367, 1267, 1036, $804 \mathrm{~cm}^{-1} ;{ }^{1} \mathrm{H}-\mathrm{NMR}\left(\mathrm{CDCl}_{3}\right) \delta 6.80(1 \mathrm{H}, \mathrm{d}$, $J=8.0 \mathrm{~Hz}, \mathrm{H}-5$ ') 6.67 (1H, d, $\left.J=1.8 \mathrm{~Hz}, \mathrm{H}-2^{\prime}\right), 6.64(1 \mathrm{H}, \mathrm{dd}, J=8.0,1.8 \mathrm{~Hz}, \mathrm{H}-6$ '), 3.85 (3H, $\left.\mathrm{s},-\mathrm{OCH}_{3}\right), 2.86-2.63(4 \mathrm{H}, \mathrm{m}, \mathrm{H}-1,-2), 2.36(2 \mathrm{H}, \mathrm{t}, J=7.4 \mathrm{~Hz}, \mathrm{H}-4), 1.58-1.51(2 \mathrm{H}, \mathrm{m}, \mathrm{H}-5), 1.24$ $(8 \mathrm{H}, \mathrm{m}, \mathrm{H}-6 \sim 9), 0.88(3 \mathrm{H}, \mathrm{t}, J=6.2 \mathrm{~Hz}, \mathrm{H}-10) ;{ }^{13} \mathrm{C}-\mathrm{NMR}\left(\mathrm{CDCl}_{3}\right) \delta 210.6,146.3,143.8,133.1,120.7$, 114.3, 111.0, 55.8, 44.6, 43.1, 31.6, 29.5, 29.1, 29.0, 23.8, 22.5, 14.0; EIMS m/z (rel. int.) $278\left(\mathrm{M}^{+}\right.$, 67), 179 (21), 151 (23), 137 (100), 117 (19), 99 (23), 55 (21); HREIMS m/z 278.1883 [M] $]^{+}$(Calcd for $\left.\mathrm{C}_{17} \mathrm{H}_{26} \mathrm{O}_{3}, 278.1881\right)$.

[7]-Paradol (5c): colorless syrup (81\%) [23]; UV (MeOH) $\lambda_{\max }(\log \varepsilon) 282$ (3.45) nm; IR (neat) $v_{\max }$ 3543, 2930, 2858, 1707, 1608, 1516, 1452, 1365, 1269, 1034, $806 \mathrm{~cm}^{-1} ;{ }^{1} \mathrm{H}-\mathrm{NMR}\left(\mathrm{CDCl}_{3}\right) \delta 6.77$ $\left(1 \mathrm{H}, \mathrm{d}, J=8.0 \mathrm{~Hz}, \mathrm{H}-5^{\prime}\right), 6.66\left(1 \mathrm{H}, \mathrm{d}, J=1.8 \mathrm{~Hz}, \mathrm{H}-2^{\prime}\right), 6.61(1 \mathrm{H}, \mathrm{dd}, J=8.0,1.8 \mathrm{~Hz}, \mathrm{H}-6$ '), 5.48 $(1 \mathrm{H}$, br s, $-\mathrm{OH}), 3.79\left(3 \mathrm{H}, \mathrm{s},-\mathrm{OCH}_{3}\right), 2.83-2.61(4 \mathrm{H}, \mathrm{m}, \mathrm{H}-1,-2), 2.33(2 \mathrm{H}, \mathrm{t}, J=7.2 \mathrm{~Hz}, \mathrm{H}-4)$, 1.58-1.51 (2H, m, H-5), $1.22(10 \mathrm{H}, \mathrm{m}, \mathrm{H}-6 \sim 10), 0.85(3 \mathrm{H}, \mathrm{t}, J=6.8 \mathrm{~Hz}, \mathrm{H}-11) ;{ }^{13} \mathrm{C}-\mathrm{NMR}\left(\mathrm{CDCl}_{3}\right)$ $\delta 210.7,146.5,143.9,132.9,120.6,114.4,111.1,55.7,44.4,42.9,31.7,29.4,29.2,29.1,29.0,23.7$, 22.5, 14.0; EIMS m/z (rel. int.) $292\left(\mathrm{M}^{+}, 36\right), 179$ (17), 151 (21), 137 (100), 119 (10), 55 (11).

[8]-Paradol (5d): colorless powder (77\%), mp 42-43 ${ }^{\circ} \mathrm{C}\left(\right.$ lit. $\left.42-43{ }^{\circ} \mathrm{C}\right)$ [23]; UV (MeOH) $\lambda_{\max }(\log \varepsilon)$ $282(3.46) \mathrm{nm}$; IR (KBr) $v_{\max } 3541,2920,2856,1707,1608,1514,1365,1271,1030,806 \mathrm{~cm}^{-1}$; ${ }^{1} \mathrm{H}-\mathrm{NMR}\left(\mathrm{CDCl}_{3}\right) \delta 6.82\left(1 \mathrm{H}, \mathrm{d}, J=7.8 \mathrm{~Hz}, \mathrm{H}-5\right.$ '), $6.68-6.63\left(2 \mathrm{H}, \mathrm{m}, \mathrm{H}-2\right.$ ', -6 '), $3.86\left(3 \mathrm{H}, \mathrm{s},-\mathrm{OCH}_{3}\right)$, 2.87-2.64 (4H, m, H-1, -2), 2.37 (2H, t, $J=7.2 \mathrm{~Hz}, \mathrm{H}-4), 1.58-1.51(2 \mathrm{H}, \mathrm{m}, \mathrm{H}-5), 1.25$ (12H, m, $\mathrm{H}-6 \sim 11), 0.88(3 \mathrm{H}, \mathrm{t}, J=6.8 \mathrm{~Hz}, \mathrm{H}-12) ;{ }^{13} \mathrm{C}-\mathrm{NMR}\left(\mathrm{CDCl}_{3}\right) \delta 210.6,146.4,143.8,133.1,120.7,114.3$, 111.0, 55.8, 44.6, 43.1, 31.8, 29.5, 29.3 (×3), 29.2, 23.8, 22.6, 14.1; EIMS $m / z$ (rel. int.) $306\left(\mathrm{M}^{+}, 17\right)$, 292 (12), 164 (21), 179 (19), 151 (22), 137 (100), 57 (10); Anal. Calcd. for $\mathrm{C}_{19} \mathrm{H}_{30} \mathrm{O}_{3}$ : C, 74.50\%; H, 9.80\%; Found: C, 74.57\%; H, 9.84\%.

[9]-Paradol (5e): colorless powder $(80 \%), \mathrm{mp} 49-50{ }^{\circ} \mathrm{C}$ (lit. $\left.48-49{ }^{\circ} \mathrm{C}\right)$ [23]; $\mathrm{UV}(\mathrm{MeOH}) \lambda_{\max }(\log \varepsilon)$ $282(3.41) \mathrm{nm}$; IR (KBr) $v_{\max } 3516,2922,2856,1712,1608,1516,1361,1273,1165,1028,856 \mathrm{~cm}^{-1}$; ${ }^{1} \mathrm{H}-\mathrm{NMR}\left(\mathrm{CDCl}_{3}\right) \delta 6.81\left(1 \mathrm{H}, \mathrm{d}, J=8.0 \mathrm{~Hz}, \mathrm{H}-5\right.$ '), $6.68-6.63\left(2 \mathrm{H}, \mathrm{m}, \mathrm{H}-2\right.$ ', -6 '), $3.86\left(3 \mathrm{H}, \mathrm{s},-\mathrm{OCH}_{3}\right)$, 2.86-2.64 (4H, m, H-1, -2), $2.36(2 \mathrm{H}, \mathrm{t}, J=7.2 \mathrm{~Hz}, \mathrm{H}-4), 1.58-1.51(2 \mathrm{H}, \mathrm{m}, \mathrm{H}-5), 1.24$ (14H, m, $\mathrm{H}-6 \sim 12), 0.87(3 \mathrm{H}, \mathrm{t}, J=6.8 \mathrm{~Hz}, \mathrm{H}-13) ;{ }^{13} \mathrm{C}-\mathrm{NMR}\left(\mathrm{CDCl}_{3}\right) \delta 210.6,146.3,143.8,133.1,120.7,114.2$, 111.0, 56.0, 44.5, 43.1, 31.8, 29.5 (×2), 29.4 29.3, 29.2, 29.1, 23.8, 22.6, 14.1; EIMS $m / z$ (rel. int.) $320\left(\mathrm{M}^{+}, 80\right), 179$ (19), 151 (21), 137 (100), 119 (8); Anal. Calcd. for $\mathrm{C}_{20} \mathrm{H}_{32} \mathrm{O}_{3}$ : C, 75.00\%; H, 10.00\%; Found: C, 75.01\%; H, 10.01\%. 
[10]-Paradol (5f): colorless powder (79\%), mp 50-51 ${ }^{\circ} \mathrm{C}$ (lit. 50-51 ${ }^{\circ} \mathrm{C}$ ) [23]; UV (MeOH) $\lambda_{\max }$ $(\log \varepsilon) 280(3.44) \mathrm{nm}$; IR (KBr) $v_{\max } 3486,2920,2856,1707,1608,1512,1361,1273,1165,1028$, $856 \mathrm{~cm}^{-1} ;{ }^{1} \mathrm{H}-\mathrm{NMR}\left(\mathrm{CDCl}_{3}\right) \delta 6.81(1 \mathrm{H}, \mathrm{d}, J=8.0 \mathrm{~Hz}, \mathrm{H}-5$ '), $6.68-6.63(2 \mathrm{H}, \mathrm{m}, \mathrm{H}-2$ ', -6 '), 3.85 (3H, $\left.\mathrm{s},-\mathrm{OCH}_{3}\right), 2.86-2.64(4 \mathrm{H}, \mathrm{m}, \mathrm{H}-1,-2), 2.37(2 \mathrm{H}, \mathrm{t}, J=7.2 \mathrm{~Hz}, \mathrm{H}-4), 1.58-1.51(2 \mathrm{H}, \mathrm{m}, \mathrm{H}-5), 1.25$ $(16 \mathrm{H}, \mathrm{m}, \mathrm{H}-6 \sim 13), 0.88(3 \mathrm{H}, \mathrm{t}, J=6.6 \mathrm{~Hz}, \mathrm{H}-14) ;{ }^{13} \mathrm{C}-\mathrm{NMR}\left(\mathrm{CDCl}_{3}\right) \delta 210.6,146.4,143.8,133.0$, 120.6, 114.3, 111.0, 55.7, 44.5, 43.0, 31.8, $29.5(\times 2), 29.429 .3(\times 2), 29.2,29.1,23.7,22.6,14.0$; Anal. Calcd. for $\mathrm{C}_{21} \mathrm{H}_{34} \mathrm{O}_{3}$ : C, 75.45\%; H, 10.18\%; Found: C, 75.49\%; H, 10.13\%.

\subsubsection{General Procedure for the Synthesis of $[n]$-Dehydroparadols $(\mathbf{6 a}-\mathbf{f})$}

A solution of $[n]$-dehydroshogaols $(\mathbf{2 a}-\mathbf{f})(0.96 \mathrm{mmol})$ in ethyl acetate $(20 \mathrm{~mL})$ containing palladium-charcoal $(5 \%, 0.015 \mathrm{~g})$ was stirred under hydrogen at atmospheric pressure and room temperature for $40 \mathrm{~min}$. The reaction mixture was monitored using thin layer chromatography (TLC) until no starting material remained. The catalyst was removed through celite, and the filtrate was concentrated under reduced pressure conditions. The product was isolated using silica gel column chromatography $($ EtOAc/hexanes $=1 / 3)$.

[5]-Dehydroparadol (6a): colorless powder (80\%), mp 52-53 ${ }^{\circ} \mathrm{C}$ (lit. 52-53 $\left.{ }^{\circ} \mathrm{C}\right)$ [23]; UV (MeOH) $\lambda_{\max }(\log \varepsilon) 340$ (4.16), 224 (3.79) nm; IR (KBr) $v_{\max }$ 3400, 2930, 2860, 1666, 1587, 1514, 1460, 1375, 1276, 1033, 979, $812 \mathrm{~cm}^{-1} ;{ }^{1} \mathrm{H}-\mathrm{NMR}\left(\mathrm{CDCl}_{3}\right) \delta 7.48(1 \mathrm{H}, \mathrm{d}, J=16.1 \mathrm{~Hz}, \mathrm{H}-1), 7.09(1 \mathrm{H}, \mathrm{dd}, J=8.1$, $2.0 \mathrm{~Hz}, \mathrm{H}-6$ ') 7.05 (1H, d, $J=2.0 \mathrm{~Hz}, \mathrm{H}-2$ ') 6.92 (1H, d, $J=8.1 \mathrm{~Hz}, \mathrm{H}-5$ '), 6.59 (1H, d, $J=16.1 \mathrm{~Hz}$, $\mathrm{H}-2), 6.12(1 \mathrm{H}$, br s, $-\mathrm{OH}), 3.92\left(3 \mathrm{H}, \mathrm{s},-\mathrm{OCH}_{3}\right), 2.64$ (2H, t, $\left.J=7.1 \mathrm{~Hz}, \mathrm{H}-4\right), 1.70-1.59$ (2H, m, H-5), 1.41-1.22 (6H, m, H-6 8), $0.88(3 \mathrm{H}, \mathrm{t}, J=6.5 \mathrm{~Hz}, \mathrm{H}-9) ;{ }^{13} \mathrm{C}-\mathrm{NMR}\left(\mathrm{CDCl}_{3}\right) \delta 200.8,148.1,146.8$, 142.6, 127.1, 124.1, 123.3, 114.8, 109.4, 55.9, 40.7, 31.6, 29.0, 24.5, 22.5, 14.0; EIMS m/z (rel. int.) $262\left(\mathrm{M}^{+}, 31\right), 192(34), 177$ (100), 145 (22), 137 (44), 117 (10), 89 (12).

[6]-Dehydroparadol (6b): colorless powder (76\%), mp 47-48 ${ }^{\circ} \mathrm{C}$ (lit. 44-45 ${ }^{\circ} \mathrm{C}$ ) [23]; $\mathrm{UV}(\mathrm{MeOH})$ $\lambda_{\max }(\log \varepsilon) 341$ (4.04), 224 (3.85) nm; IR (KBr) $v_{\max }$ 3400, 2926, 2856, 1666, 1601, 1514, 1460, 1375, 1278, 1031, 979, $810 \mathrm{~cm}^{-1} ;{ }^{1} \mathrm{H}-\mathrm{NMR}\left(\mathrm{CDCl}_{3}\right) \delta 7.47(1 \mathrm{H}, \mathrm{d}, J=16.0 \mathrm{~Hz}, \mathrm{H}-1), 7.08(1 \mathrm{H}, \mathrm{dd}, J=8.1$, $1.8 \mathrm{~Hz}, \mathrm{H}-6$ ') 7.04 (1H, d, $J=1.8 \mathrm{~Hz}, \mathrm{H}-2$ ') $6.91(1 \mathrm{H}, \mathrm{d}, J=8.1 \mathrm{~Hz}, \mathrm{H}-5$ '), $6.58(1 \mathrm{H}, \mathrm{d}, J=16.0 \mathrm{~Hz}$, $\mathrm{H}-2), 6.19(1 \mathrm{H}$, br s, $-\mathrm{OH}), 3.90\left(3 \mathrm{H}, \mathrm{s},-\mathrm{OCH}_{3}\right), 2.63$ (2H, t, $\left.J=7.2 \mathrm{~Hz}, \mathrm{H}-4\right), 1.69-1.59$ (2H, m, H-5), 1.31-1.27 (8H, m, H-6 9), $0.88(3 \mathrm{H}, \mathrm{t}, J=6.6 \mathrm{~Hz}, \mathrm{H}-10) ;{ }^{13} \mathrm{C}-\mathrm{NMR}\left(\mathrm{CDCl}_{3}\right) \delta 200.8,148.1,146.8$, $142.6,126.9,123.9,123.3,114.8,109.4,55.9,40.6,31.6,29.3,29.0,24.5,22.5,14.0$; EIMS $\mathrm{m} / \mathrm{z}$ (rel. int.) $276\left(\mathrm{M}^{+}, 31\right), 192$ (40), 177 (100), 145 (19), 137 (71), 117 (10), 89 (11), 55(10); HREIMS $m / z 276.1727[\mathrm{M}]^{+}$(Calcd for $\mathrm{C}_{17} \mathrm{H}_{24} \mathrm{O}_{3}, 276.1725$ ).

[7]-Dehydroparadol (6c): colorless powder (75\%), mp 49-50 ${ }^{\circ} \mathrm{C}$ (lit. 45-46 $\left.{ }^{\circ} \mathrm{C}\right)$ [23]; UV (MeOH) $\lambda_{\max }(\log \varepsilon) 338$ (4.03), $225(3.80) \mathrm{nm}$; IR (KBr) $v_{\max }$ 3401, 2926, 2854, 1676, 1589, 1514, 1460, 1377, 1207, 1033, 979, $810 \mathrm{~cm}^{-1} ;{ }^{1} \mathrm{H}-\mathrm{NMR}\left(\mathrm{CDCl}_{3}\right) \delta 7.46(1 \mathrm{H}, \mathrm{d}, J=16.1 \mathrm{~Hz}, \mathrm{H}-1), 7.05(1 \mathrm{H}, \mathrm{dd}, J=8.0$, $1.8 \mathrm{~Hz}, \mathrm{H}-6$ ') 7.01 (1H, d, $J=1.8 \mathrm{~Hz}, \mathrm{H}-2$ ') 6.89 (1H, d, $J=8.0 \mathrm{~Hz}, \mathrm{H}-5$ '), 6.57 (1H, d, $J=16.1 \mathrm{~Hz}$, $\mathrm{H}-2), 3.86\left(3 \mathrm{H}, \mathrm{s},-\mathrm{OCH}_{3}\right), 2.61(2 \mathrm{H}, \mathrm{t}, J=7.2 \mathrm{~Hz}, \mathrm{H}-4), 1.67-1.57$ (2H, m, H-5), 1.26-1.23 (10H, m, $\mathrm{H}-6 \sim 10), 0.85(3 \mathrm{H}, \mathrm{t}, J=6.8 \mathrm{~Hz}, \mathrm{H}-11) ;{ }^{13} \mathrm{C}-\mathrm{NMR}\left(\mathrm{CDCl}_{3}\right) \delta 200.9,148.3,146.9,142.8,126.8,123.8$, 123.3, 114.9, 109.5, 55.8, 40.5, 31.7, $29.3(\times 2), 29.1,24.5,22.6,14.0$; EIMS m/z (rel. int.) 290 
(M+15), 205 (12), 192 (28), 177 (63), 137 (100), 91 (11), 55(12); HREIMS m/z 290.1885 [M] ${ }^{+}$(Calcd for $\mathrm{C}_{18} \mathrm{H}_{26} \mathrm{O}_{3}, 290.1881$ ).

[8]-Dehydroparadol (6d): colorless powder (73\%), mp 58-59 ${ }^{\circ} \mathrm{C}$ (lit. 57-58 $\left.{ }^{\circ} \mathrm{C}\right)$ [23]; $\mathrm{UV}(\mathrm{MeOH})$ $\lambda_{\max }(\log \varepsilon) 339$ (4.09), $223(3.96) \mathrm{nm}$; IR (KBr) $v_{\max }$ 3401, 2925, 2854, 1675, 1589, 1514, 1460, 1272, 1033, 979, $810 \mathrm{~cm}^{-1} ;{ }^{1} \mathrm{H}-\mathrm{NMR}\left(\mathrm{CDCl}_{3}\right) \delta 7.48(1 \mathrm{H}, \mathrm{d}, J=16.0 \mathrm{~Hz}, \mathrm{H}-1), 7.06(1 \mathrm{H}, \mathrm{dd}, J=8.0$, $1.8 \mathrm{~Hz}, \mathrm{H}-6$ ') 7.04 (1H, d, $J=1.8 \mathrm{~Hz}, \mathrm{H}-2$ '), 6.91 (1H, d, $J=8.0 \mathrm{~Hz}, \mathrm{H}-5$ '), 6.59 (1H, d, $J=16.0 \mathrm{~Hz}$, H-2), 6.19 (1H, br s, -OH), $3.91\left(3 \mathrm{H}, \mathrm{s},-\mathrm{OCH}_{3}\right), 2.63$ (2H, t, $\left.J=7.0 \mathrm{~Hz}, \mathrm{H}-4\right), 1.67-1.62$ (2H, m, H-5), $1.28-1.25(12 \mathrm{H}, \mathrm{m}, \mathrm{H}-6 \sim 11), 0.86(3 \mathrm{H}, \mathrm{t}, J=6.8 \mathrm{~Hz}, \mathrm{H}-12) ;{ }^{13} \mathrm{C}-\mathrm{NMR}\left(\mathrm{CDCl}_{3}\right) \delta 200.8,148.1,146.8$, 142.7, 127.0, 123.9, 123.3, 114.8, 109.4, 55.9, 40.6, 31.8, $29.4(\times 2), 29.3,29.2$, 24.5, 22.6, 14.0; EIMS m/z (rel. int.) 304 (M+27), 205 (13), 192 (34), 177 (66), 151 (18), 137 (100), 91 (10), 55(12); Anal. Calcd for $\mathrm{C}_{19} \mathrm{H}_{28} \mathrm{O}_{3}$ : C, 75.00\%; H, 9.21\%; Found: C, 74.99\%; H, 9.25\%.

[9]-Dehydroparadol (6e): colorless powder (74\%), mp 56-58 ${ }^{\circ} \mathrm{C}$ (lit. 53-54 ${ }^{\circ} \mathrm{C}$ ) [23]; $\mathrm{UV}(\mathrm{MeOH})$ $\lambda_{\max }(\log \varepsilon) 337$ (3.96), $224(3.74) \mathrm{nm}$; IR (KBr) $v_{\max }$ 3395, 2925, 2854, 1666, 1589, 1516, 1460, 1277, 1033, 979, $812 \mathrm{~cm}^{-1} ;{ }^{1} \mathrm{H}-\mathrm{NMR}\left(\mathrm{CDCl}_{3}\right) \delta 7.47(1 \mathrm{H}, \mathrm{d}, J=16.0 \mathrm{~Hz}, \mathrm{H}-1), 7.08(1 \mathrm{H}, \mathrm{dd}, J=8.0$, $1.8 \mathrm{~Hz}, \mathrm{H}-6$ ') 7.03 (1H, d, $J=1.8 \mathrm{~Hz}, \mathrm{H}-2$ ') 6.90 (1H, d, $J=8.0 \mathrm{~Hz}, \mathrm{H}-5$ '), 6.58 (1H, d, $J=16.0 \mathrm{~Hz}$, $\mathrm{H}-2), 6.22(1 \mathrm{H}$, br s, $-\mathrm{OH}), 3.90\left(3 \mathrm{H}, \mathrm{s},-\mathrm{OCH}_{3}\right), 2.63$ (2H, t, $\left.J=7.2 \mathrm{~Hz}, \mathrm{H}-4\right), 1.69-1.62$ (2H, m, H-5), $1.28-1.24(14 \mathrm{H}, \mathrm{m}, \mathrm{H}-6 \sim 12), 0.86(3 \mathrm{H}, \mathrm{t}, J=6.6 \mathrm{~Hz}, \mathrm{H}-13) ;{ }^{13} \mathrm{C}-\mathrm{NMR}\left(\mathrm{CDCl}_{3}\right) \delta 200.8,148.1,146.8$, 142.6, 126.9, 123.9, 123.3, 114.8, 109.4, 55.8, 40.6, 31.8, 29.5, 29.4, $29.3(\times 2), 29.2,24.5,22.5,14.0$; EIMS m/z (rel. int.) $318\left(\mathrm{M}^{+}, 27\right), 192$ (57), 177 (100), 153 (22), 137 (39), 55(23).

[10]-Dehydroparadol (6f): colorless powder (79\%), mp 72-73 ${ }^{\circ} \mathrm{C}$ (lit. $76-77{ }^{\circ} \mathrm{C}$ ) [23]; UV (MeOH) $\lambda_{\max }(\log \varepsilon) 339$ (3.99), $225(3.78) \mathrm{nm}$; IR(KBr) $v_{\max } 3412,2920,2854,1666,1589,1512,1460,1277$, $1033 \mathrm{~cm}^{-1} ;{ }^{1} \mathrm{H}-\mathrm{NMR}\left(\mathrm{CDCl}_{3}\right) \delta 7.47(1 \mathrm{H}, \mathrm{d}, J=16.0 \mathrm{~Hz}, \mathrm{H}-1), 7.10-7.04(2 \mathrm{H}, \mathrm{m}, \mathrm{H}-2$ ', 6'), 6.89 (1H, d, $\left.J=8.2 \mathrm{~Hz}, \mathrm{H}-5^{\prime}\right), 6.58(1 \mathrm{H}, \mathrm{d}, J=16.0 \mathrm{~Hz}, \mathrm{H}-2), 6.25(1 \mathrm{H}$, br s, $-\mathrm{OH}), 3.90\left(3 \mathrm{H}, \mathrm{s},-\mathrm{OCH}_{3}\right)$, $2.63(2 \mathrm{H}, \mathrm{t}, J=7.2 \mathrm{~Hz}, \mathrm{H}-4), 1.69-1.59$ (2H, m, H-5), 1.28-1.25 (16H, m, H-6 13), 0.86 (3H, t, $J=6.4 \mathrm{~Hz}, \mathrm{H}-14) ;{ }^{13} \mathrm{C}-\mathrm{NMR}\left(\mathrm{CDCl}_{3}\right) \delta 200.8,148.1,146.8,142.6,126.9,123.9,123.3,114.8,109.4$, $55.8,40.5,31.8,29.5(\times 2), 29.4,29.3(\times 2), 29.2,24.5,22.6,14.0$.

\subsubsection{General Procedure for the Synthesis of $[n]$-Gingerols $(\mathbf{8 a}-\mathbf{f})$}

A solution of $[n]$-dehydrogingerols $(3 \mathbf{a}-\mathbf{f})(1.1 \mathrm{mmol})$ in ethyl acetate $(20 \mathrm{~mL})$ containing palladium-charcoal $(5 \%, 0.04 \mathrm{~g})$ was stirred under hydrogen at atmospheric pressure and room temperature for $40 \mathrm{~min}$. The reaction mixture was monitored using TLC until no starting material remained. The catalyst was removed through celite, and the filtrate was concentrated under reduced pressure. The product was isolated using silica gel column chromatography $($ EtOAc/hexanes $=1 / 2)$.

[5]-Gingerol (8a): colorless powder (85\%), mp 44-45 ${ }^{\circ} \mathrm{C}$ (lit. $\left.45-46{ }^{\circ} \mathrm{C}\right)$; UV (MeOH) $\lambda_{\max }(\log \varepsilon)$ 282 (3.44), 224 (3.85) nm; IR (KBr) $v_{\max }$ 3460, 2943, 2864, 1704, 1612, 1138, 1371, 1271, 1138, 1034, $806 \mathrm{~cm}^{-1} ;{ }^{1} \mathrm{H}-\mathrm{NMR}\left(\mathrm{CDCl}_{3}\right) \delta 6.78\left(1 \mathrm{H}, \mathrm{d}, J=7.8 \mathrm{~Hz}, \mathrm{H}-5\right.$ '), $6.64\left(1 \mathrm{H}, \mathrm{d}, J=1.8 \mathrm{~Hz}, \mathrm{H}-2^{\prime}\right)$, $6.61(1 \mathrm{H}, \mathrm{dd}, J=7.8,1.8 \mathrm{~Hz}, \mathrm{H}-6$ '), $4.34(1 \mathrm{H}, \mathrm{br} \mathrm{s},-\mathrm{OH}), 4.06-3.94(1 \mathrm{H}, \mathrm{m}, \mathrm{H}-5), 3.81(3 \mathrm{H}, \mathrm{s}$, $\left.-\mathrm{OCH}_{3}\right), 2.48-2.65(4 \mathrm{H}, \mathrm{m}, \mathrm{H}-1,-2), 2.52-2.48(2 \mathrm{H}, \mathrm{m}, \mathrm{H}-4), 1.50-1.22$ (6H, m, H-6 8), 0.86 (3H, t, 
$J=7.0 \mathrm{~Hz}, \mathrm{H}-9) ;{ }^{13} \mathrm{C}-\mathrm{NMR}\left(\mathrm{CDCl}_{3}\right) \delta 211.4,147.0,144.0,132.6,120.7,114.4,111.0,67.6,55.9$, 49.3, 45.4, 36.1, 29.2, 27.6, 22.6, 14.3; EIMS m/z (rel. int.) $280\left(\mathrm{M}^{+}, 31\right), 205$ (9), 150 (50), 137 (100), 91 (10); HREIMS $m / z 280.1677$ [M] $]^{+}$(Calcd for $\mathrm{C}_{16} \mathrm{H}_{24} \mathrm{O}_{4}, 280.1674$ ).

[6]-Gingerol (8b): colorless syrup (84\%); UV (MeOH) $\lambda_{\max }(\log \varepsilon) 282$ (3.51) nm; IR (KBr) $v_{\max }$ 3469, 2937, 2860, 1705, 1608, 1516, 1371, 1140, 1036, $806 \mathrm{~cm}^{-1} ;{ }^{1} \mathrm{H}-\mathrm{NMR}\left(\mathrm{CDCl}_{3}\right) \delta 6.79(1 \mathrm{H}, \mathrm{d}$, $J=7.8 \mathrm{~Hz}, \mathrm{H}-5$ '), $6.66-6.60$ (2H,m, H-2', -6'), 4.05-3.99 (1H, m, H-5), $3.83\left(3 \mathrm{H}, \mathrm{s},-\mathrm{OCH}_{3}\right)$, 2.86-2.66 (4H, m, H-1, -2), 2.53-2.48 (2H, m, H-4), 1.49-1.24 (8H, m, H-6 9), 0.86 (3H, t, $J=6.2 \mathrm{~Hz}$, $\mathrm{H}-10) ;{ }^{13} \mathrm{C}-\mathrm{NMR}\left(\mathrm{CDCl}_{3}\right) \delta 211.4,146.4,143.9,132.5,120.6,114.4,110.9,67.6,55.7,49.2,45.3$, 36.3, 31.6, 29.1, 25.6, 22.5, 14.0; EIMS m/z (rel. int.) $294\left(\mathrm{M}^{+}, 18\right), 205$ (7), 194 (14), 150 (40), 137 (100), 91 (11); HREIMS m/z 294.1831 [M] (Calcd for $\mathrm{C}_{17} \mathrm{H}_{26} \mathrm{O}_{4}, 294.1831$ ).

[7]-Gingerol (8c): colorless powder (86\%), mp 101-102 ${ }^{\circ} \mathrm{C}$; UV (MeOH) $\lambda_{\max }(\log \varepsilon) 281$ (3.67), $223(3.95) \mathrm{nm}$; IR (KBr) $v_{\max } 3524,2926,2858,1705,1608,1516,1369,1271,1036,806 \mathrm{~cm}^{-1}$; ${ }^{1} \mathrm{H}-\mathrm{NMR}\left(\mathrm{CDCl}_{3}\right) \delta 6.80(1 \mathrm{H}, \mathrm{d}, J=8.0 \mathrm{~Hz}, \mathrm{H}-5$ ') $, 6.66-6.60(2 \mathrm{H}, \mathrm{m}, \mathrm{H}-2$ ', -6 ' $), 4.06-3.95(1 \mathrm{H}, \mathrm{m}$, $\mathrm{H}-5), 3.84$ (3H, s, $\left.-\mathrm{OCH}_{3}\right), 2.86-2.66$ (4H, m, H-1, -2), 2.52-2.48 (2H, m, H-4), 1.51-1.25 (10H, m, $\mathrm{H}-6 \sim 10), 0.86(3 \mathrm{H}, \mathrm{t}, J=6.6 \mathrm{~Hz}, \mathrm{H}-11) ;{ }^{13} \mathrm{C}-\mathrm{NMR}\left(\mathrm{CDCl}_{3}\right) \delta 211.5,146.5,144.0,132.6,120.7,114.5$, 111.1, 67.7, 55.8, 49.3, 45.4, 36.5, 31.8, 29.2, 29.1, 25.4, 22.6, 14.1; EIMS $m / z$ (rel. int.) $308\left(\mathrm{M}^{+}, 16\right)$, 290 (15), 205 (21), 150 (32), 137 (100), 91 (13), 55 (24); Anal. Calcd. for $\mathrm{C}_{18} \mathrm{H}_{28} \mathrm{O}_{4}$ : C, 70.12\%; H, 9.09\%; Found: C, 70.14\%; H, 9.04\%.

[8]-Gingerol (8d): colorless syrup (83\%); UV (MeOH) $\lambda_{\max }(\log \varepsilon) 282$ (3.63), 222 (3.93) nm; IR (neat) $v_{\max } 3516,2928,2858,1705,1608,1516,1452,1271,1035,806 \mathrm{~cm}^{-1} ;{ }^{1} \mathrm{H}-\mathrm{NMR}\left(\mathrm{CDCl}_{3}\right) \delta 6.80$ $\left(1 \mathrm{H}, \mathrm{d}, J=7.8 \mathrm{~Hz}, \mathrm{H}-5\right.$ '), $6.66-6.61\left(2 \mathrm{H}, \mathrm{m}, \mathrm{H}-2\right.$ ', -6 '), 4.05-3.95 (1H, m, H-5), 3.85 (3H, s, $\left.-\mathrm{OCH}_{3}\right)$, 2.86-2.67 (4H, m, H-1, -2), 2.53-2.48 (2H, m, H-4), 1.49-1.25 (12H, m, H-6 11), 0.86 (3H, t, $J=6.2 \mathrm{~Hz}, \mathrm{H}-12) ;{ }^{13} \mathrm{C}-\mathrm{NMR}\left(\mathrm{CDCl}_{3}\right) \delta 211.3,146.4,143.9,132.5,120.6,114.3,110.9,67.6,55.7$, 49.2, 45.3, 36.4, 31.7, 29.4, 29.1, 25.3, 22.5, 14.0; EIMS m/z (rel. int.) $322\left(\mathrm{M}^{+}, 38\right), 150$ (50), 137 (100), 55 (9).

[9]-Gingerol (8e): colorless syrup (84\%); UV (MeOH) $\lambda_{\max }(\log \varepsilon) 281$ (3.33), 224 (3.71) nm; IR (neat) $v_{\max } 3535,2924,2856,1704,1608,1514,1369,1271,1031,804 \mathrm{~cm}^{-1} ;{ }^{1} \mathrm{H}-\mathrm{NMR}\left(\mathrm{CDCl}_{3}\right) \delta 6.80$ $\left(1 \mathrm{H}, \mathrm{d}, J=7.8 \mathrm{~Hz}, \mathrm{H}-5\right.$ '), $6.66-6.60$ (2H, m, H-2', -6'), 4.07-3.99 (1H, m, H-5), 3.84 (3H, s, $\left.-\mathrm{OCH}_{3}\right)$, 2.86-2.66 (4H, m, H-1, -2), 2.53-2.48 (2H, m, H-4), 1.38-1.25 (14H, m, H-6 12), 0.87 (3H, t, $J=6.8 \mathrm{~Hz}, \mathrm{H}-13) ;{ }^{13} \mathrm{C}-\mathrm{NMR}\left(\mathrm{CDCl}_{3}\right) \delta 211.4,146.5,143.9,132.6,120.7,114.5,111.0,67.7,55.8,49.3$, 45.4, 36.5, 31.8, $29.5(\times 2), 29.2(\times 2), 25.4,22.6,14.0$; EIMS m/z (rel. int.) $336\left(\mathrm{M}^{+}, 32\right), 318(14), 205$ (17), 150 (36), 137 (100), 55 (9); HREIMS m/z: 336.2300 [M] $]^{+}$(Calcd for $\mathrm{C}_{20} \mathrm{H}_{32} \mathrm{O}_{4}, 336.2300$ ).

[10]-Gingerol (8f): colorless syrup (85\%); UV (MeOH) $\lambda_{\max }(\log \varepsilon) 282$ (3.43), 224 (3.75) nm; IR (neat) $v_{\max } 3439,2920,2854,1706,1608,1514,1369,1271,1031,804 \mathrm{~cm}^{-1} ;{ }^{1} \mathrm{H}-\mathrm{NMR}\left(\mathrm{CDCl}_{3}\right) \delta 6.78$ $\left(1 \mathrm{H}, \mathrm{d}, J=7.8 \mathrm{~Hz}, \mathrm{H}-5\right.$ '), 6.65-6.59 (2H, m, H-2', -6'), 4.05-3.99 (1H, m, H-5), 3.82 (3H, s, $\left.-\mathrm{OCH}_{3}\right)$, 2.83-2.65 (4H, m, H-1, -2), 2.53-2.49 (2H, m, H-4), 1.38-1.25 (16H, m, H-6 13), 0.87 (3H, t, $J=6.6 \mathrm{~Hz}, \mathrm{H}-14) ;{ }^{13} \mathrm{C}-\mathrm{NMR}\left(\mathrm{CDCl}_{3}\right) \delta 211.3,146.4,143.8,132.4,120.5,114.4,110.9,67.6,55.6$, $49.2,45.2,36.3,31.7,29.4(\times 3), 29.2,29.1,25.3,22.5,14.0$. 


\subsubsection{General Procedure for the Synthesis of $[n]-$ Shogaols $(\mathbf{4 a}-\mathbf{f})$}

Conc. $\mathrm{HCl}(0.1 \mathrm{~mL})$ was added dropwise to a solution of $[n]$-gingerols $(\mathbf{8 a}-\mathbf{f})(0.54 \mathrm{mmol})$ in acetone $(10 \mathrm{~mL})$ at room temperature. The reaction mixture was stirred for $15 \mathrm{~min}$ and then cooled to $0{ }^{\circ} \mathrm{C}$ in an ice bath, neutralized by saturated sodium bicarbonate, and extracted with $\mathrm{CH}_{2} \mathrm{Cl}_{2}(3 \times 10 \mathrm{~mL})$. The organic layers were combined, washed with brine, dried over $\mathrm{MgSO}_{4}$, and concentrated under reduced pressure. The crude product was diluted with acetone $(10 \mathrm{~mL})$ and then potassium carbonate $(0.81 \mathrm{mmol})$ was added at room temperature. The reaction mixture was stirred for $6 \mathrm{~h}$, then cooled to $0{ }^{\circ} \mathrm{C}$ in an ice bath, neutralized by $5 \% \mathrm{HCl}_{(\mathrm{aq})}$, and extracted with $\mathrm{CH}_{2} \mathrm{Cl}_{2}(3 \times 10 \mathrm{~mL})$. The organic layers were combined, washed with brine, dried over $\mathrm{MgSO}_{4}$, and concentrated under reduced pressure. The product was isolated using silica gel column chromatography (ethyl acetate/hexanes $=1 / 3$ ).

[5]-Shogaol (4a): yellow syrup (86\%) [24]; UV (MeOH) $\lambda_{\max }(\log \varepsilon) 281$ (3.51), 225 (4.31) nm; IR (neat) $v_{\max } 3451,2932,2862,1685,1629,1514,1456,1271,1034,984,806 \mathrm{~cm}^{-1} ;{ }^{1} \mathrm{H}-\mathrm{NMR}\left(\mathrm{CDCl}_{3}\right) \delta$ 6.89-6.65 (4H, m, H-2', -5', -6', -5), 6.07 (1H, dt, $J=15.8,1.6 \mathrm{~Hz}, \mathrm{H}-4), 5.50$ (1H, br s, -OH), 3.87 $\left(3 \mathrm{H}, \mathrm{s},-\mathrm{OCH}_{3}\right), 2.87-2.79$ (4H, m, H-1, -2), 2.25-2.14 (2H, m, H-6), 1.49-1.23 (4H, m, H-7, -8), 0.90 $(3 \mathrm{H}, \mathrm{t}, J=6.8 \mathrm{~Hz}, \mathrm{H}-9) ;{ }^{13} \mathrm{C}-\mathrm{NMR}\left(\mathrm{CDCl}_{3}\right) \delta 199.8,147.8,146.3,143.8,133.2,130.3,120.8,114.3$, 111.1, 55.8, 42.0, 32.1, 30.1, 29.9, 22.2, 13.8; EIMS m/z (rel. int.) 262 ( $\left.\mathrm{M}^{+}, 46\right), 205$ (42), 151 (16), 137 (100), 55 (22).

[6]-Shogaol (4b): yellow syrup (85\%) [24]; UV (MeOH) $\lambda_{\max }(\log \varepsilon) 282$ (3.47), 224 (4.25) nm; IR (neat) $v_{\max } 3424,2928,2860,1662,1616,1514,1456,1271,1034,982,808 \mathrm{~cm}^{-1} ;{ }^{1} \mathrm{H}-\mathrm{NMR}\left(\mathrm{CDCl}_{3}\right) \delta$ 6.89-6.65 (4H, m, H-2', -5', -6', -5), 6.08 (1H, dt, $J=16.0,1.4 \mathrm{~Hz}, \mathrm{H}-4), 5.54$ (1H, br s, -OH), 3.86 $\left(3 \mathrm{H}, \mathrm{s},-\mathrm{OCH}_{3}\right), 2.89-2.79$ (4H, m, H-1, -2), 2.24-2.13 (2H, m, H-6), 1.51-1.26 (6H, m, H-7 9), 0.88 $(3 \mathrm{H}, \mathrm{t}, J=6.5 \mathrm{~Hz}, \mathrm{H}-10) ;{ }^{13} \mathrm{C}-\mathrm{NMR}\left(\mathrm{CDCl}_{3}\right) \delta 199.8,147.8,146.3,143.8,133.2,130.2,120.7,114.2$, 111.0, 55.8, 41.9, 32.4, 31.3, 29.8, 27.1, 22.4, 13.9; EIMS m/z (rel. int.) $276\left(\mathrm{M}^{+}, 43\right), 205$ (52), 151 (16), 137 (100), 119 (10), 55 (18).

[7]-Shogaol (4c): yellow syrup (83\%) [24]; UV (MeOH) $\lambda_{\max }(\log \varepsilon) 282$ (3.44), 225 (4.18) nm; IR (neat) $v_{\max } 3450,2927,2856,1691,1626,1516,1460,1367,1271,1036,978,815 \mathrm{~cm}^{-1} ;{ }^{1} \mathrm{H}-\mathrm{NMR}$ $\left(\mathrm{CDCl}_{3}\right) \delta$ 6.89-6.65 (4H, m, H-2', -5', -6', -5), $6.08(1 \mathrm{H}, \mathrm{dt}, J=16.0,1.5 \mathrm{~Hz}, \mathrm{H}-4), 3.86(3 \mathrm{H}$, $\left.\mathrm{s},-\mathrm{OCH}_{3}\right), 2.86-2.82(4 \mathrm{H}, \mathrm{m}, \mathrm{H}-1,-2), 2.24-2.14(2 \mathrm{H}, \mathrm{m}, \mathrm{H}-6), 1.47-1.26$ (8H, m, H-7 10), 0.88 (3H, $\mathrm{t}, J=6.7 \mathrm{~Hz}, \mathrm{H}-11) ;{ }^{13} \mathrm{C}-\mathrm{NMR}\left(\mathrm{CDCl}_{3}\right) \delta 199.8,147.9,146.4,143.8,133.2,130.3,120.8,114.3$, 111.1, 55.8, 42.0, 32.5, 31.5, 29.8, 28.8, 28.0, 22.5, 14.0; EIMS $m / z$ (rel. int.) $290\left(\mathrm{M}^{+}, 27\right), 205$ (28), $151(14), 137(100), 55(9)$.

[8]-Shogaol (4d): yellow syrup (79\%) [24]; UV (MeOH) $\lambda_{\max }(\log \varepsilon) 282$ (3.72), 225 (4.52) nm; IR (neat) $v_{\max } 3433,2926,2856,1675,1629,1514,1456,1271,1034,980,808 \mathrm{~cm}^{-1} ;{ }^{1} \mathrm{H}-\mathrm{NMR}\left(\mathrm{CDCl}_{3}\right)$ $\delta$ 6.89-6.65 (4H, m, H-2', -5', -6', -5), $6.08(1 \mathrm{H}, \mathrm{dt}, J=15.8,1.6 \mathrm{~Hz}, \mathrm{H}-4), 3.86\left(3 \mathrm{H}, \mathrm{s},-\mathrm{OCH}_{3}\right)$, 2.85-2.82 (4H, m, H-1, -2), 2.24-2.13 (2H, m, H-6), 1.47-1.27 (10H, m, H-7 11), 0.88 (3H, t, $J=6.7 \mathrm{~Hz}, \mathrm{H}-12) ;{ }^{13} \mathrm{C}-\mathrm{NMR}\left(\mathrm{CDCl}_{3}\right) \delta 199.8,147.9,146.4,143.8,133.2,130.2,120.7,114.3,111.1$, 55.8, 41.9, 32.4, 31.7, 29.8, 29.1, 28.0, 22.6, 14.0; EIMS m/z (rel. int.) $304\left(\mathrm{M}^{+}, 34\right), 205$ (51), 151 (18), 137 (100), 69 (20), 55 (26). 
[9]-Shogaol (4e): yellow syrup (85\%) [24]; UV (MeOH) $\lambda_{\max }(\log \varepsilon) 282$ (3.55), 226 (4.36) nm; IR (neat) $v_{\max } 3432,2926,2856,1685,1638,1514,1471,1271,1034,982,808 \mathrm{~cm}^{-1} ;{ }^{1} \mathrm{H}-\mathrm{NMR}\left(\mathrm{CDCl}_{3}\right)$ $\delta$ 6.91-6.66 (4H, m, H-2', -5', -6', -5), $6.08(1 \mathrm{H}, \mathrm{dt}, J=15.7,1.4 \mathrm{~Hz}, \mathrm{H}-4), 3.87$ (3H, s, $\left.-\mathrm{OCH}_{3}\right)$, 2.87-2.80 (4H, m, H-1, -2), 2.25-2.14 (2H, m, H-6), 1.47-1.27 (12H, m, H-7 12), 0.88 (3H, t, $J=6.6 \mathrm{~Hz}, \mathrm{H}-13) ;{ }^{13} \mathrm{C}-\mathrm{NMR}\left(\mathrm{CDCl}_{3}\right) \delta 199.8,147.9,146.4,143.9,133.2,130.3,120.8,114.3,111.1$, 55.9, 42.0, 32.5, 31.8, 29.9, 29.3, 29.1, 28.1, 22.6, 14.1; EIMS m/z (rel. int.) 318 (M , 35), 205 (58), 151 (16), 137 (100), 55 (15).

[10]-Shogaol (4f): yellow syrup (88\%) [24]; UV (MeOH) $\lambda_{\max }(\log \varepsilon) 284$ (3.45), 225 (4.16) nm; IR (neat) $v_{\max } 3513,2924,2852,1688,1638,1512,1471,1271,1034,982,808 \mathrm{~cm}^{-1} ;{ }^{1} \mathrm{H}-\mathrm{NMR}\left(\mathrm{CDCl}_{3}\right)$ $\delta$ 6.89-6.64 (4H, m, H-2', -5', -6', -5), $6.08(1 \mathrm{H}, \mathrm{dt}, J=15.6,1.4 \mathrm{~Hz}, \mathrm{H}-4), 3.85\left(3 \mathrm{H}, \mathrm{s},-\mathrm{OCH}_{3}\right)$, 2.84-2.82 (4H, m, H-1, -2), 2.19-2.13 (2H, m, H-6), 1.43-1.25 (14H, m, H-7 13), 0.87 (3H, t, $J=6.4 \mathrm{~Hz}, \mathrm{H}-14) ;{ }^{13} \mathrm{C}-\mathrm{NMR}\left(\mathrm{CDCl}_{3}\right) \delta 199.9,147.9,146.4,143.9,133.2,130.3,120.7,114.3,111.1$, $55.8,41.9,32.5,31.8,29.8,29.4,29.3,29.2,29.1,28.1,22.6,14.1$.

3.2.8. General Procedure for the Synthesis of $[n]$-Isodehydrogingerdiones (1a-f)

DMSO $(0.15 \mathrm{~mL}, 2.16 \mathrm{mmol})$ was added dropwise to a solution of oxalyl chloride $(0.12 \mathrm{~mL}$, $1.40 \mathrm{mmol})$ in acetone $(10 \mathrm{~mL})$ at $-50-60{ }^{\circ} \mathrm{C}$ under argon. The reaction mixture was stirred for $3 \mathrm{~min}$, and then a solution of $[n]$-dehydrogingerols $(\mathbf{3 a}-\mathbf{f})(1.08 \mathrm{mmol})$ in $\mathrm{CH}_{2} \mathrm{Cl}_{2}(5 \mathrm{~mL})$ was slowly added. The reaction mixture was stirred for another $15 \mathrm{~min}, \mathrm{Et}_{3} \mathrm{~N}$ was added to the mixture; the temperature was changed to $0{ }^{\circ} \mathrm{C}$ in an ice bath for $20 \mathrm{~min}$, and the reaction mixture was then neutralized using $5 \%$ $\mathrm{HCl}_{(\mathrm{aq})}$ and extracted with $\mathrm{CH}_{2} \mathrm{Cl}_{2}(3 \times 10 \mathrm{~mL})$. The organic layers were combined, washed with brine, dried over $\mathrm{MgSO}_{4}$, and concentrated under reduced pressure. The product [ $n$ ]-isodehydrogingerdiones (1a-f) and $[n]$-dehydroshogaols (2a-f) were isolated using silica gel column chromatography (ethyl acetate/hexanes $=1 / 3)$.

[5]-Isodehydrogingerdione (1a): yellow syrup (51\%); UV (MeOH) $\lambda_{\max }(\log \varepsilon) 369$ (4.39), 255 (3.71) nm; IR (KBr) $v_{\max } 3358,2958,2866,1634,1576,1512,1427,1273,1030,966,837 \mathrm{~cm}^{-1}$; ${ }^{1} \mathrm{H}-\mathrm{NMR}$ $\left(\mathrm{CDCl}_{3}\right) \delta 7.51(1 \mathrm{H}, \mathrm{d}, J=15.8 \mathrm{~Hz}, \mathrm{H}-1), 7.07(1 \mathrm{H}, \mathrm{dd}, J=8.2,1.8 \mathrm{~Hz}, \mathrm{H}-6$ ' $), 7.00(1 \mathrm{H}, \mathrm{d}, J=1.8 \mathrm{~Hz}$, H-2'), 6.90 (1H, d, $J=8.2 \mathrm{~Hz}, \mathrm{H}-5$ '), 6.34 (1H, d, $J=15.8 \mathrm{~Hz}, \mathrm{H}-2), 5.62$ (1H, s, H-4), 3.91 (3H, $\left.\mathrm{s},-\mathrm{OCH}_{3}\right), 2.38(2 \mathrm{H}, \mathrm{t}, J=7.2 \mathrm{~Hz}, \mathrm{H}-6), 1.70-1.58(2 \mathrm{H}, \mathrm{m}, \mathrm{H}-7), 1.46-1.28(2 \mathrm{H}, \mathrm{m}, \mathrm{H}-8), 0.92$ (3H, t, $J=7.2 \mathrm{~Hz}, \mathrm{H}-9) ;{ }^{13} \mathrm{C}-\mathrm{NMR}\left(\mathrm{CDCl}_{3}\right) \delta 200.2,178.0,147.6,146.8,139.8,127.6,122.6,120.5,114.8$, $109.4,100.1,55.9,39.8,27.7,22.4,13.8$.

[6]-Isodehydrogingerdione (1b): yellow syrup (49\%); UV (MeOH) $\lambda_{\max }(\log \varepsilon) 369$ (4.37), 256 (3.70) nm; IR (KBr) $v_{\max } 3418,2956,2864,1634,1591,1512,1427,1271,1032,970,816 \mathrm{~cm}^{-1}$; ${ }^{1} \mathrm{H}-\mathrm{NMR}$ $\left(\mathrm{CDCl}_{3}\right) \delta 7.51(1 \mathrm{H}, \mathrm{d}, J=15.8 \mathrm{~Hz}, \mathrm{H}-1), 7.06(1 \mathrm{H}, \mathrm{dd}, J=8.0,1.8 \mathrm{~Hz}, \mathrm{H}-6$ ' $), 7.01(1 \mathrm{H}, \mathrm{d}, J=1.8 \mathrm{~Hz}$, H-2'), 6.90 (1H, d, $J=8.0 \mathrm{~Hz}, \mathrm{H}-5$ ') $6.34(1 \mathrm{H}, \mathrm{d}, J=15.8 \mathrm{~Hz}, \mathrm{H}-2), 5.61(1 \mathrm{H}, \mathrm{s}, \mathrm{H}-4), 3.94(3 \mathrm{H}$, s, $\left.-\mathrm{OCH}_{3}\right), 2.37(2 \mathrm{H}, \mathrm{t}, J=7.4 \mathrm{~Hz}, \mathrm{H}-6), 1.69-1.57(2 \mathrm{H}, \mathrm{m}, \mathrm{H}-7), 1.35-1.28$ (2H, m, H-8 9), 0.90 (3H, $\mathrm{t}, J=6.2 \mathrm{~Hz}, \mathrm{H}-10) ;{ }^{13} \mathrm{C}-\mathrm{NMR}\left(\mathrm{CDCl}_{3}\right) \delta 200.2,178.0,147.6,146.7,139.8,127.7,122.6,120.5$, 114.8, 109.4, 100.1, 55.9, 40.1, 31.4, 25.3, 22.4, 13.9. 
[7]-Isodehydrogingerdione (1c): yellow syrup (59\%); UV (MeOH) $\lambda_{\max }(\log \varepsilon) 368$ (4.17), 257 (3.62) nm; IR $(\mathrm{KBr}) v_{\max } 3423,2928,2858,1634,1582,1512,1427,1273,1031,974,814 \mathrm{~cm}^{-1}$; ${ }^{1} \mathrm{H}-\mathrm{NMR}$ $\left(\mathrm{CDCl}_{3}\right) \delta 7.52(1 \mathrm{H}, \mathrm{d}, J=15.8 \mathrm{~Hz}, \mathrm{H}-1), 7.08(1 \mathrm{H}, \mathrm{dd}, J=8.2,1.8 \mathrm{~Hz}, \mathrm{H}-6$ ') $7.02(1 \mathrm{H}, \mathrm{d}, J=1.8 \mathrm{~Hz}$, H-2'), 6.91 (1H, d, $J=8.2 \mathrm{~Hz}, \mathrm{H}-5$ '), 6.33 (1H, d, $J=15.8 \mathrm{~Hz}, \mathrm{H}-2), 5.94$ (1H, br s, -OH), 5.62 (1H, s, $\mathrm{H}-4), 3.93\left(3 \mathrm{H}, \mathrm{s},-\mathrm{OCH}_{3}\right), 2.37(2 \mathrm{H}, \mathrm{t}, J=7.6 \mathrm{~Hz}, \mathrm{H}-6), 1.64-1.61(2 \mathrm{H}, \mathrm{m}, \mathrm{H}-7), 1.30-1.24(6 \mathrm{H}, \mathrm{m}$, $\mathrm{H}-8 \sim 10), 0.89(3 \mathrm{H}, \mathrm{t}, J=6.6 \mathrm{~Hz}, \mathrm{H}-11) ;{ }^{13} \mathrm{C}-\mathrm{NMR}\left(\mathrm{CDCl}_{3}\right) \delta 200.2,178.0,147.7,146.8,139.8,127.7$, $122.6,120.5,114.8,109.5,100.1,55.9,40.1,31.6,29.0,25.6,22.5,14.0$.

[9]-Isodehydrogingerdione (1e): yellow syrup (48\%); UV (MeOH) $\lambda_{\max }(\log \varepsilon) 369$ (4.37), 254 (3.72) nm; IR (KBr) $v_{\max } 3423,2955,2854,1634,1583,1512,1427,1271,1031,970,816 \mathrm{~cm}^{-1} ;{ }^{1} \mathrm{H}-\mathrm{NMR}\left(\mathrm{CDCl}_{3}\right)$ $\delta 7.52(1 \mathrm{H}, \mathrm{d}, J=15.6 \mathrm{~Hz}, \mathrm{H}-1), 7.08$ (1H, dd, $J=8.2,1.8 \mathrm{~Hz}, \mathrm{H}-6$ '), 7.01 (1H, d, $J=1.8 \mathrm{~Hz}, \mathrm{H}-2$ '), $6.91(1 \mathrm{H}, \mathrm{d}, J=8.2 \mathrm{~Hz}, \mathrm{H}-5$ '), $6.34(1 \mathrm{H}, \mathrm{d}, J=15.6 \mathrm{~Hz}, \mathrm{H}-2), 5.91(1 \mathrm{H}, \mathrm{br} \mathrm{s},-\mathrm{OH}), 5.62(1 \mathrm{H}, \mathrm{s}, \mathrm{H}-4)$, $3.93\left(3 \mathrm{H}, \mathrm{s},-\mathrm{OCH}_{3}\right), 2.37(2 \mathrm{H}, \mathrm{t}, J=7.2 \mathrm{~Hz}, \mathrm{H}-6), 1.67-1.60(2 \mathrm{H}, \mathrm{m}, \mathrm{H}-7), 1.29-1.27(10 \mathrm{H}, \mathrm{m}$, $\mathrm{H}-8 \sim 12), 0.88(3 \mathrm{H}, \mathrm{t}, J=6.2 \mathrm{~Hz}, \mathrm{H}-13) ;{ }^{13} \mathrm{C}-\mathrm{NMR}\left(\mathrm{CDCl}_{3}\right) \delta 200.1,177.9,147.6,146.7,140.0,127.6$, $122.5,120.5,114.7,109.4,100.1,55.9,40.1,31.8,29.3,29.1,25.6,22.6,14.0$.

\subsection{Antiplatelet Aggregatory Bioassay}

An assay of the antiplatelet aggregatory activity of the isolated compound was conducted according to the procedures of Teng and coworkers $[25,26]$. Washed platelets were prepared from blood withdrawn with a siliconized syringe from the marginal vein of New Zealand rabbits. The platelet suspension was obtained from EDTA-anticoagulated platelet-rich plasma according to the washing procedure described previously. The platelet number was determined using a cell counter (Hema-laser 2, Sebia, France) and adjusted to $3.0 \times 10^{8}$ platelets $/ \mathrm{mL}$. The platelet pellets were suspended in Tyrode's solution containing $\mathrm{Ca}^{2+}(1 \mathrm{mM})$ and bovine serum albumin $(0.35 \%)$. All glassware was siliconized. Platelet aggregation was measured using the turbidimetric method [26]. The aggregations were measured with a Lumi-aggregometer (Model 1020, Payton, Canada) connected to two dual-channel recorders.

\section{Conclusions}

Eight groups of derivatives based on the skeletons of shogaol and gingerol, the active pungent principles from ginger, were synthesized and evaluated for their antiplatelet bioactivity. Among the compounds synthesized, [6]-paradol $\mathbf{5 b}$ displayed the most significant inhibition of platelet aggregation induced by AA. Anti-PAF induced platelet aggregation activity was not found in the present study, suggesting that [6]-paradol $\mathbf{5 b}$ is a selective inhibitor. The traditional use of Z. officinale is to promote the blood circulation necessary for removing blood stasis, and the results of this study substantiated the anti-platelet aggregation activity of these synthetic derivatives related to shogaol and gingerol. It is valuable to explore new anti-platelet aggregation drugs based on the skeleton of $[n]$-paradol or other principles reported from the Zingiber series.

\section{Acknowledgments}

The authors wish to express appreciation to the National Science Council, Taiwan, ROC, for financial support of the present research. 


\section{Conflicts of Interest}

The authors declare no conflicts of interest.

\section{References}

1. Tang, W.; Eisenbrand, G. Chinese Drugs of Plant Origin; Springer-Verlag: Berlin, Germany, 1992; pp. 1011-1105.

2. Kikuzaki, H.; Nakatani, N. Antioxidant effects of some ginger constituents. J. Food. Sci. 1993, $58,1407-1410$.

3. Hasheimi, S.R.; Zulkifli, I.; Somchit, M.N.; Zunita, Z.; Loh, T.C.; Soleimani, A.F.; Tang, S.C. Dietary supplementation of Zingiber officinale and Zingiber zerumbet to heat-stressed broiler chickens and its effect on heat shock protein 70 expression, blood parameters and body temperature. J. Anim. Physiol. Anim. Nutr. (Berl.) 2013, 97, 632-638.

4. Koo, K.L.; Ammit, A.J.; Tran, V.H.; Duke, C.C.; Roufoqalis, B.D. Gingerols and related analogues inhibit arachidonic acid-induced human platelet serotonin release and aggregation. Thromb. Res. 2001, 103, 387-397.

5. Nurtjahja-Tjendraputra, E.; Ammit, A.J.; Roufogalis, B.D.; Tran, V.H.; Duke, C.C. Effective anti-platelet and COX-1 enzyme inhibitors from pungent constituents of ginger. Thromb. Res. 2003, 111, 259-265.

6. Pancho, L.R.; Kimura, I.; Unno, R.; Kurono, M.; Kimura, M. Reversed effects between crude and processed ginger extracts on $\mathrm{PGF}_{2 \alpha}$-induced contraction in mouse mesenteric veins. Jpn. J. Pharmacol. 1989, 50, 243-246.

7. Kiuchi, F.; Shibuya, M.; Sankawa, U. Inhibitors of prostaglandin biosynthesis from ginger. Chem. Pharm. Bull. 1982, 30, 754-757.

8. Kuwada, T.; Sakabe, S.I.; Watanabe, T.; Yamamoto, M.; Iwai, K. Some pungent principles of spices cause the adrenal medulla to secrete catecholamine in anesthetized rats. Proc. Soc. Exp. Boil. Med. 1988, 188, 229-233.

9. Chen, C.Y.; Cheng, K.C.; Chang, A.Y.; Lin, Y.T.; Hseu, Y.C.; Wang, H.M. 10-Shogaol, an Antioxidant from Zingiber officinale for skin cell proliferation and migration enhancer. Int. J. Mol. Sci. 2012, 13, 1762-1777.

10. Huang, Q.R.; Iwamoto, M.; Aoki, S.; Tanaka, N.; Tajima, K.; Yamahara, J.; Takaishi, Y.; Yoshida, M.; Tomimatsu, T.; Tamai, Y. Anti-5-hydroxytryptamine 3 effect of galanolactone, diterpenoid isolated from ginger. Chem. Pharm. Bull. 1991, 39, 397-399.

11. Shoji, N.; Iwasa, A.; Takemoto, T.; Ishida, Y.; Ohizumi, Y. Cardiotonic principles of ginger (Zingiber officinale Roscoe). J. Pharm. Sci. 1982, 71, 1174-1175.

12. Adewunmi, C.O.; Oquntimein, B.O.; Furu, P. Molluscicidal and antischistosomal activities of Zingiber officinale. Planta Med. 1990, 56, 374-376.

13. Liu, Y.; Whelan, R.J.; Pattnaik, B.R.; Ludwig, K.; Subudhi, E.; Rowland, H.; Claussen, N.; Zucker, N.; Uppal, S.; Kushner, D.M.; et al. Terpenoids from Zingiber officinale (Ginger) induce apoptosis in endometrial cancer cells through the activation of p53. PLoS One 2012, 7, e53178. 
14. Yamahara, J.; Hatakeyama, S.; Taniguchi, K.; Kawamura, M.; Yoshikawa, M. Stomachic principles in ginger. II. Pungent and anti-ulcer effects of low polar constituents isolated from ginger, the dried rhizoma of Zingiber officinale Roscoe cultivated in Taiwan. The absolute stereostructure of a new diarylheptanoid. Yakugaku Zasshi 1992, 112, 645-655.

15. Kikuzaki, H.; Usuguchi, J.; Nakatani, N. Constitutents of Zingiberaceae. I. diarylheptanoids from the rhizomes of ginger (Zingiber officinale Roscoe). Chem. Pharm. Bull. 1991, 39, 120-122.

16. Murata, T.; Shinohara, M.; Miyamoto, M. Isolation of hexahydrocurcumin, dihydrogingerol and two additional pungent principles from ginger. Chem. Pharm. Bull. 1972, 20, 2291-2292.

17. Yoshikawa, M.; Hatakeyama, S.; Taniguchi, K.; Matuda, H.; Yamahara, J. 6-Gingesulfonic acid, a new anti-ulcer principle, and gingerglycolipids $\mathrm{A}, \mathrm{B}$, and $\mathrm{C}$, three new monoacyldigalactosyl-glycerols, from Zingiberis rhizoma originating in Taiwan. Chem. Pharm. Bull. 1992, 40, 2239-2241.

18. Danyer, C.V.; Jachson, P.; Loakes, D.M.; Ellis, M.R.; Young, D.A.B. Isolation of antirhinoviral sesquiterpenes from ginger (Zingiber officinale). J. Nat. Prod. 1994, 57, 658-662.

19. Wender, P.A.; Jesudason, C.D.; Nakahira, H.; Tamura, N.; Tebbe, A.L.; Ueno, Y. The first synthesis of a daphnane diterpene: The enantiocontrolled total synthesis of $(+)$-resiniferatoxin. J. Am. Chem. Soc. 1997, 119, 12976-12977.

20. Liao, Y.R.; Leu, Y.L.; Chan, Y.Y.; Kuo, P.C.; Wu, T.S. Anti-platelet aggregation and vasorelaxing effects of the constituents of the rhizomes of Zingiber officiale. Molecules 2012, 17, 8928-8927.

21. Wu, T.S.; Chern, H.J.; Damu, A.G.; Kuo, P.C.; Su, C.R.; Lee, E.J.; Teng, C.M. Flavonoids and andrographolides from Andrographis paniculata and their antiplatelet aggregatory and vasorelaxing effects. J. Asian Nat. Prod. Res. 2008, 10, 17-24.

22. Denniff, P.; Macleod, I.; Whiting, D.A. Syntheses of the $( \pm)-[n]$-gingerols (pungent principles of ginger) and related compounds through regioselective aldol condensations: Relative pungency assays. J. Chem. Soc. Perkin Trans. 1981, 1, 82-87.

23. Locksley, H.D.; Rainey, D.K. Pungent compounds. Part I. An improved synthesis of the paradols (alkyl 4-hydroxy-3-methoxyphenthyl ketones) and an assessment of their pungency. J. Chem. Soc. Perkin Trans. 1972, 1, 3001-3006.

24. Kim, D.S.H.L.; Kim, J.Y. Side-chain length is important for shogaols in protecting neuronal cells from beta-amyloid insult. Bioorg. Med. Chem. Lett. 2004, 14, 1287-1289.

25. Teng, C.M.; Chen, W.C.; Ko, F.N.; Lee, L.G.; Huang, T.F.; Chen, Y.P.; Hsu, H.Y. Two antiplatelet agents from Magnolia officinalis. Thromb. Res. 1988, 50, 757-765.

26. Born, G.V.R.; Cross, M.J. Effects of inorganic ions and of plasma proteins on the aggregation of blood platelets by adenosine diphosphate. J. Physiol. 1963, 168, 178-195.

Sample Availability: Samples of all the synthetic compounds are available from the authors.

(C) 2014 by the authors; licensee MDPI, Basel, Switzerland. This article is an open access article distributed under the terms and conditions of the Creative Commons Attribution license (http://creativecommons.org/licenses/by/3.0/). 